\title{
The Physical Meaning of the Wave Function
}

\author{
Ilija Barukčić \\ Internist, Horand Strasse, Jever, Germany \\ Email:Barukcic@t-online.de
}

Received 25 April 2016; accepted 5 June 2016; published 8 June 2016

Copyright (C) 2016 by author and Scientific Research Publishing Inc.

This work is licensed under the Creative Commons Attribution International License (CC BY). http://creativecommons.org/licenses/by/4.0/

(c) $\underset{\mathrm{EY}}{\mathrm{E}}$ Open Access

\begin{abstract}
Under some well-defined conditions, the mathematical formalism of quantum mechanics enables physicists, chemists and others to calculate and predict the outcome of a vast number of experiments. In fact, especially the Schrödinger equation which involves an imaginary quantity describes how a quantum state of a physical system changes with time and is one of the main pillars of modern quantum mechanics. The wave function itself is a determining part of the Schrödinger equation, but the physical meaning of the wave function is still not clear. Altogether, does the wave function represent a new kind of reality? This publication will solve the problem of the physical meaning of the wave function by investigating the relationship between the wave function and the theory of special relativity. It is shown that the wave function is determined by notion co-ordinate time of the special theory of relativity. Moreover, the result of this investigation suggests a new understanding of the wave function, according to which the wave function and co-ordinate time of the theory of special relativity are equivalent.
\end{abstract}

\section{Keywords}

Quantum Theory, Relativity Theory, Unified Field Theory, Causality

\section{Introduction}

The Schrödinger equation, published in 1926 by the Austrian physicist Erwin Rudolf Josef Alexander Schrödinger (1887-1961), is determined by Newton's (1642-1727) second law, in its original form known as "Mutationem motus proportionalem esse vi motrici impressae, et fieri secundum lineam rectam qua vis illa imprimitur" [1] and to some extent an analogue of Newton's second law in quantum mechanics. Leonhard Euler (1707-1783), a pioneering Swiss mathematician and physicist, formulated in 1752 Newton lexsecunda [2] in its mathematical form as

$$
{ }_{O} \vec{F}={ }_{O} m \times{ }_{O} \vec{a} .
$$

The famous Schrödinger equation [3], a partial differential equation, describes how a quantum state of a sys- 
tem changes with time. The Schrödinger equation for any system, no matter whether relativistic or not, no matter how complicated, has the form

$$
{ }_{R} H \times{ }_{R} \Psi\left({ }_{R} X,{ }_{R} t\right)=i \hbar \frac{\partial}{\partial t}{ }_{R} \Psi\left({ }_{R} X,{ }_{R} t\right)
$$

where $i$ is the imaginary unit, $\hbar=\frac{h}{2 \times \pi}$ is Planck's constant divided by $2 \times \pi$, the symbol $\frac{\partial}{\partial t}$ indicates a partial derivative with respect to time ${ }_{R} t,{ }_{R} \Psi\left({ }_{R} X\right.$, $\left.{ }_{R} t\right)$ is the wave function of the quantum system, and ${ }_{R} H$ is the Hamiltonian operator. In quantum mechanics, the Hamiltonian operator is a quantum mechanical operator which characterizes the total energy of a quantum mechanical system and is usually denoted by ${ }_{R} H$. The Hamiltonian operator ${ }_{R} H$ takes different forms depending upon situation.

The form of the Schrödinger equation itself depends on the physical situation and is determined by the wavefunction ${ }_{R} \Psi\left({ }_{R} X,{ }_{R} t\right)$. The wave function itself is one of the most fundamental concepts of quantum mechanics. Schrödinger himself states that he has "not attached a definite physical meaning to the wavefunction..." [3].

The physical meaning of the wave function is in dispute in the alternative interpretations of quantum mechanics. The de Broglie-Bohm theory or the many-worlds interpretation has another view on the physical meaning of the wave function then the Copenhagen interpretation of the wave function. In view of this very unsatisfactory situation, it seems to be necessary to put some light on the problem of the physical meaning of the wave function from the standpoint of the theory of special relativity. In a similar way, hereafter, we shall restrict ourselves to a one-dimensional treatment in order to decrease the amount of notation needed, since in all cases, the generalization to four (i.e. quantum mechanics) or n-dimensions (i.e. quantum field theory) will be equally simple.

\section{Material and Methods}

\subsection{Definitions}

\section{Definition: Proof by contradiction (Reductio ad Absurdum)}

The logical background of a proof by contradiction is Aristotle's law of non-contradiction. A rigorous proof by contradiction of a theorem follows the standard method of contradiction used in science and mathematics and should be convincing as much as possible. For the first, we assume that a claim/a theorem/a proposition/a statement et cetera which has to be proved, is true. One then proceeds to demonstrate that a conclusion drawn from such a claim/a theorem/a proposition/a statement et cetera leads to a contradiction. Hence, the supposed claim/ theorem/proposition/statement et cetera is deemed to be false.

Consequently, we are then led to conclude that it was wrong to assume the claim/the theorem/the proposition/ the statement was true. Thus far, the claim/the theorem/the proposition/the statement is proved to be false.

\section{Definition: Thought experiments}

Properly constructed (real or) thought experiments (as devices of scientific investigation) can be used for diverse reasons in a variety of areas. Thought experiments can help us to investigate some basic properties of nature even under conditions when it is too difficult or too expensive to run a real experiment. Furthermore, a thought experiment can provide some evidence against or in favour of a theory. However, a thought experiment is not a substitute for a real experiment.

\subsubsection{Newton's Axiomata sive leges motus}

Newton's laws of motion, verified by experiment and observation over centuries, are valid in inertial reference frames. However, any reference frame moving in uniform motion with respect to an inertial frame is an inertial frame too. In quantum mechanics concepts such as force, momentum, and position are defined as linear operators which operate on a quantum state. Newton's three physical laws, the foundation of classical mechanics, have been expressed in several different ways and can be summarized as follows.

\section{Definition: Newton's first law}

"Lex. I. Corpus omne perseverare in statu suo quiescendi vel movendi uniformmiter in directum, nisi quatenus a viribus impressis cogitur statum illum mutare" [1].

Newton's first translated to English reads:

"Law. I. Every body persists in its state of being at rest or of moving uniformly straight forward, except insofar as it is compelled to change its state by force impressed." 
Newton's first law first law states that if the net force (a vector sum of all forces acting on something) is zero, then the velocity, a vector (speed and direction of motion) quantity, of this something is constant. Newton's first law can be stated mathematically as

$$
\sum_{t=0}^{n} \overrightarrow{{ }_{t}} \vec{F}=0 .
$$

\section{Definition: Newton's second law}

Newton defined his second law as:

“Lex. II. Mutationem motuspro portionalem esse vi motrici impressae, \& fieri se-cundum lineam rectam qua vis illa imprimitur” [1].

Newton's second translated quite closely to English reads:

"Law. II. The alteration of motion is ever proportional to the motive force impressed; and is made in the direction of the right line in which that force is impressed."

In general, Newton's second law is stated mathematically as

$$
\overrightarrow{{ }_{R} F}={ }_{R} m \times \overrightarrow{{ }_{R} a} \text {. }
$$

Thus far, something at rest will stay at rest unless a net force acts upon it and equally something in motion will stay in motion (will not change its velocity) unless a net force acts upon it. In other words, if something is accelerating, then there must be a net force acting on it.

\section{Definition: Newton's third law}

Newton defined his own third law as:

"Lex. III. Actioni contrariam semper et aequalem esse reactionem: sive corporum duorum actiones in se mutuo semper esse æquales et in partes contrarias dirigi” [1].

Newton's third law (actio = reactio) translated to English reads:

"Law. III. To every action there is always opposed an equal reaction: or the mutual actions of two bodies upon each other are always equal, and directed to contrary parts."

The two forces, equal but opposite with ${ }_{O} F$ called the "action" and ${ }_{R} F$ the "reaction" are part of every single interaction. It does not matter which force is called the reaction and which force is called the action. Newton's third law is stated mathematically as

$$
\overrightarrow{{ }_{O} F}=-\overline{{ }_{R} F} \text {. }
$$

\subsubsection{Special Theory of Relativity}

Definition: The relativistic energy ${ }_{R} E$ (of a system)

In general, it is

$$
{ }_{R} E={ }_{R} m \times c^{2}
$$

where ${ }_{R} E$ denotes the total ("relativistic") energy of a system, ${ }_{R} m$ denotes the "relativistic" mass and $c$ denotes the speed of the light in vacuum.

Scholium.

Einstein defined the matter/mass-energy equivalence as follows:

“Gibt ein Körper die Energie $L$ in Form von Strahlung ab, so verkleinert sich seine Masse um $L / V^{2} \ldots$ Die Masse eines Körpers ist ein Maß für dessen Energieinhalt” [4].

In other words, due to Einstein, energy and mass are equivalent.

"Eines der wichtigsten Resultate der Relativitätstheorie ist die Erkenntnis, daß jegliche Energie E eine ihr proportionale Trägheit $\left(E / c^{2}\right)$ besitzt” [5].

It is equally correct by Einstein to point out, matter/mass and energy are equivalent.

"Da Masse und Energie nach den Ergebnissen der speziellen Relativitätstheorie das Gleiche sind und die Energie formal durch den symmetrischen Energietensor $\left(T_{\mu v}\right)$ beschrieben wird, so besagt dies, daß das G-Geld [gravitational field, author] durch den Energietensor der Materie bedingt und bestimmt ist” [6].

The term relativistic mass ${ }_{R} m$ was coined by Gilbert and Tolman [7].

Definition: Einstein's mass-energy equivalence relation 
The Einsteinian matter/mass-energy equivalence [4] lies at the core of today physics. In general, due to Einstein's special theory of relativity it is

$$
{ }_{O} m={ }_{R} m \times \sqrt[2]{1-\frac{v^{2}}{c^{2}}}
$$

or equally

$$
{ }_{O} E \equiv{ }_{O} m \times c^{2} \equiv{ }_{R} m \times c^{2} \times \sqrt[2]{1-\frac{v^{2}}{c^{2}}} \equiv{ }_{R} E \times \sqrt[2]{1-\frac{v^{2}}{c^{2}}}
$$

or equally

$$
\frac{{ }_{O} E}{{ }_{R} E}=\frac{{ }_{O} m \times c^{2}}{{ }_{R} m \times c^{2}}=\sqrt[2]{1-\frac{v^{2}}{c^{2}}}
$$

where ${ }_{O} E$ denotes the "rest" energy, ${ }_{O} \mathrm{~m}$ denotes the "rest" mass, ${ }_{R} E$ denotes the "relativistic" energy, ${ }_{R} m$ denotes the "relativistic" mass, $v$ denotes the relative velocity between the two observers and c denotes the speed of light in vacuum.

\section{Scholium.}

The constancy of the speed of the light $c$ is something relative and nothing absolute. If we follow Einstein, the constancy of the speed of the light $c$ is determined by a constant gravitational potential. "Dagegen bin ich der Ansicht, daß das Prinzip der Konstanz der Lichtgeschwindigkeit sich nur insoweit aufrecht erhalten läßt, als man sich auf raum-zeitliche Gebiete von konstantem Gravitationspotential beschränkt. Hier liegt nach meiner Meinung die Grenze der Gültigkeit... des Prinzips der Konstanz der Lichtgeschwindigkeit und damit unserer heutigen Relativitätstheorie” [5].

\section{Definition: Normalized relativistic energy-momentum relation}

The normalized relativistic energy momentum relation [8], a probability theory consistent formulation of Einstein's energy momentum relation, is determined as

$$
\frac{{ }_{0} m^{2}}{{ }_{R} m^{2}}+\frac{v^{2}}{c^{2}}=1
$$

while the "particle-wave-dualism" [8] is determined as

$$
\frac{{ }_{O} m^{2}}{{ }_{R} m^{2}}+\frac{v^{2}}{c^{2}} \equiv \frac{{ }_{O} m^{2} \times c^{2} \times c^{2}}{{ }_{R} m^{2} \times c^{2} \times c^{2}}+\frac{v^{2} \times{ }_{R} m^{2} \times c^{2}}{c^{2} \times{ }_{R} m^{2} \times c^{2}} \equiv \frac{{ }_{O} E^{2}}{{ }_{R} E^{2}}+\frac{{ }_{R} p^{2} \times c^{2}}{{ }_{R} E^{2}} \equiv \frac{{ }_{O} E^{2}}{{ }_{R} E^{2}}+\frac{{ }_{W} E^{2}}{{ }_{R} E^{2}} \equiv 1
$$

where ${ }_{W} E=\left({ }_{R} p \times c\right)$ denotes the energy of an electro-magnetic wave and ${ }_{R} p$ denotes the "relativistic" momentum while $c$ is the speed of the light in vacuum.

\section{Definition: The relativistic potential energy}

Following Einstein in his path of thoughts, we define the relativistic potential energy ${ }_{p} E[8]$ as

$$
{ }_{P} E \equiv \frac{{ }_{O} E \times{ }_{O} E}{{ }_{R} E} \equiv \frac{{ }_{O} E}{{ }_{R} E} \times{ }_{O} E \equiv \sqrt[2]{1-\frac{v^{2}}{c^{2}}} \times{ }_{O} E
$$

Scholium.

The definition of the relativistic potential energy ${ }_{p} E$ is supported by Einstein's publication in 1907. Einstein himself demands that there is something like a relativistic potential energy.

"Jeglicher Energie E kommt also im Gravitationsfelde eine Energie der Lage zu, die ebenso groß ist, wie die Energie der Lage einer ‘ponderablen’ Masse von der Größe E/c” [9].

Translated into English:

"Thus, to each energy $E$ in the gravitational field there corresponds an energy of position that equals the potential energy of a 'ponderable' mass of magnitude $E / c^{2}$."

The relativistic potential energy ${ }_{p} E$ can be viewed as the energy which is determined by an observer $\mathrm{P}$ which is at rest relative to the relativistic potential energy. The observer which is at rest relative to the relativistic po- 
tential energy will measure its own time, the relativistic potential time pt.

Definition: The relativistic kinetic energy (the "vis viva")

The relativistic kinetic energy ${ }_{K} \boldsymbol{E}$ is defined [8] in general as

$$
{ }_{K} E \equiv \frac{{ }_{W} E \times{ }_{W} E}{{ }_{R} E} \equiv \frac{{ }_{R} m \times v \times C_{R} \times{ }_{R} m \times v \times c}{{ }_{R} m \times c^{2}} \equiv{ }_{R} p \times v \equiv{ }_{R} m \times v^{2}
$$

where ${ }_{R} m$ denotes the "relativistic mass" and $v$ denotes the relative velocity. In general, it is

$$
{ }_{R} E \equiv{ }_{R} H \equiv{ }_{P} E+{ }_{K} E \equiv{ }_{P} H+{ }_{K} H
$$

where ${ }_{P} E$ denotes the relativistic potential energy, ${ }_{K} E$ denotes the relativistic kinetic energy, ${ }_{P} H$ denotes the Hamiltonian of the relativistic potential energy, ${ }_{K} H$ denotes the Hamiltonian of the relativistic kinetic energy. Multiplying this equation by the wave function ${ }_{R} \Psi$, we obtain a relativity consistent form of Schrödinger's equation as

$$
{ }_{R} E \times{ }_{R} \Psi \equiv{ }_{R} H \times{ }_{R} \Psi \equiv\left({ }_{P} E \times{ }_{R} \Psi\right)+\left({ }_{K} E \times{ }_{R} \Psi\right) \equiv\left({ }_{P} H \times{ }_{R} \Psi\right)+\left({ }_{K} H \times{ }_{R} \Psi\right)
$$

Scholium.

The historical background of the relativistic kinetic energy ${ }_{K} E$ is backgrounded by the long lasting and very famous dispute between Leibniz (1646-1716) and Newton (1642-1726). In fact, the core of this controversy was the dispute about the question, what is preserved through changes. Leibnitz himself claimed, that "vis viva" [10], [11] or the relativistic kineticenergy ${ }_{K} E={ }_{R} m \times v \times v$ was preserved through changes. In contrast to Leibnitz, Newton was of the opinion that the momentum ${ }_{R} p={ }_{R} m \times v$ was preserved through changes. The observer which is at rest relative to the relativistic kinetic energy will measure its own time, the relativistic kinetic time ${ }_{k}$ t.

\section{Definition: Einstein's Relativistic Time Dilation Relation}

An accurate clock in motion slows down with respect a stationary observer (observer at rest). The proper time ${ }_{o} t$ of a clock moving at constant velocity $v$ is related to a stationary observer's coordinate time ${ }_{R} t$ by Einstein's relativistic time dilation [12] and defined as

$$
{ }_{O} t={ }_{R} t \times \sqrt[2]{1-\frac{v^{2}}{c^{2}}}
$$

where ${ }_{0} t$ denotes the "proper" time, ${ }_{R} t$ denotes the "relativistic" (i.e. stationary or coordinate) time, $v$ denotes the relative velocity and c denotes the speed of light in vacuum.

Scholium.

Coordinate systems can be chosen freely, deepening upon circumstances. In many coordinate systems, an event can be specified by one time coordinate and three spatial coordinates. The time as specified by the time coordinate is denoted as coordinate time. Coordinate time is distinguished from proper time. The concept of proper time, introduced by Hermann Minkowski in 1908 and denoted as $o t$, incorporates Einstein's time dilation effect. In principle, Einstein is defining time exclusively for every place where a watch, measuring this time, is located.

“... Definition... der... Zeit... für den Ort, an welchem sich die Uhr... befindet...” [12].

In general, a watch is treated as being at rest relative to the place, where the same watch is located.

"Es werde ferner mittels der im ruhenden System befindlichen ruhenden Uhren die Zeit $t$ [i.e. ${ }_{R} t$, author] des ruhenden Systems ... bestimmt, ebensowerde die Zeit $\tau$ [ot, author] des bewegten Systems, in welchen sich relativ zu letzterem ruhende Uhren befinden, bestimmt..." [12].

Due to Einstein, it is necessary to distinguish between clocks as such which are qualified to mark the time ${ }_{R} t$ when at rest relatively to the stationary system $R$, and the time ${ }_{o} t$ when at rest relatively to the moving system $\mathrm{O}$.

"Wir denken uns ferner eine der Uhren, welche relative zum ruhenden System ruhend die Zeit $t\left[{ }_{R} t\right.$, author], relative zum bewegten System ruhend die Zeit $\tau$ [ot, author] anzugeben befähigt sind..." [12].

In other words, we have to take into account that both clocks i.e. observers have at least one point in common, the stationary observer $R$ and the moving observer $\mathrm{O}$ are at rest, but at rest relative to what? The stationary observer $R$ is at rest relative to a stationary co-ordinate system $R$, the moving observer $O$ is at rest relative to a moving co-ordinate system $O$. Both co-ordinate systems can but must not be at rest relative to each other. The time ${ }_{R} t$ of the stationary system $\mathrm{R}$ is determined by clocks which are at rest relatively to that stationary system $R$. 
Similarly, the time ${ }_{o} t$ of the moving system $O$ is determined by clocks which are at rest relatively to that the moving system $O$. In last consequence, due to Einstein's theory of special relativity, a moving clock (ot) will measure a smaller elapsed time between two events than a non-moving (inertial) clock $\left.{ }_{R} t\right)$ between the same two events.

Definition: The normalized relativistic time dilation relation

As defined above, due to Einstein's special relativity, it is

$$
{ }_{O} t={ }_{R} t \times \sqrt[2]{1-\frac{v^{2}}{c^{2}}}
$$

where ${ }_{o} t$ denotes the "proper" time, ${ }_{R} t$ denotes the "relativistic" (i.e. stationary or coordinate) time, $v$ denotes the relative velocity and $c$ denotes the speed of light in vacuum. Equally, it is

$$
\frac{{ }_{O} t}{{ }_{R} t}=\sqrt[2]{1-\frac{v^{2}}{c^{2}}}
$$

or

$$
\frac{{ }_{O} t}{c^{2}} \times \frac{c^{2}}{{ }_{R} t}=\sqrt[2]{1-\frac{v^{2}}{c^{2}}}
$$

or

$$
\frac{{ }_{o} t^{2}}{{ }_{R} t^{2}}=1-\frac{v^{2}}{c^{2}}
$$

The normalized relativistic time dilation is defined as

$$
\frac{{ }_{O} t^{2}}{{ }_{R} t^{2}}+\frac{v^{2}}{c^{2}}=1 .
$$

Definition: The relationship between ${ }_{R} t$ and ${ }_{R} g$

In general, we define the mathematical identity

$$
{ }_{R} t \equiv c^{2} \times{ }_{R} g .
$$

Scholium.

In our understanding, ${ }_{R} g$ represents the gravitational field as determined by a stationary observer R, which has to be proofed.

Definition: The relationship between $o t$ and $o g$

We define another mathematical identity

$$
{ }_{o} t \equiv c^{2} \times{ }_{o} g
$$

Scholium.

In our understanding, $o g$ represents something similar to the gravitational field as determined by a co-moving observer $\mathrm{O}$, which is at rest relative to the "rest" energy ${ }_{O} E$.

Definition: The relationship between ${ }_{W} t$ and ${ }_{R} g$

We define the following mathematical identity.

$$
{ }_{W} t \equiv v \times C \times{ }_{R} g .
$$

Scholium.

In our understanding, $w^{t}$ represents something similar to the time as associated with the electro-magnetic wave ${ }_{W} E$.

Definition: The relationship between ${ }_{K} t$ and ${ }_{R} g$

We define another mathematical identity. 


$$
{ }_{K} t \equiv \frac{{ }_{W} t \times{ }_{W} t}{{ }_{R} t} \equiv \frac{{ }_{W} t}{{ }_{R} t} \times{ }_{W} t \equiv \frac{v^{2} \times c^{2} \times{ }_{R} g^{2}}{c^{2} \times{ }_{R} g} \equiv v^{2} \times{ }_{R} g .
$$

Scholium.

The time ${ }_{K} t$ is the time as determined by an observer which is at rest relative to the relativistic kinetic energy ${ }_{K} E$.

Definition: The relationship between ${ }_{p} t$ and ${ }_{o} t$

We define the following mathematical identity.

$$
{ }_{P} t \equiv \frac{{ }_{O} t \times{ }_{O} t}{{ }_{R} t} \equiv \frac{{ }_{O} t}{{ }_{R} t} \times{ }_{O} t \equiv \sqrt[2]{1-\frac{v^{2}}{c^{2}}} \times{ }_{O} t
$$

Scholium.

The time ${ }_{p} t$ is the time as determined by an observer which is at rest relative to the relativistic potential energy ${ }_{p} E$.

Definition: The Relationship between ${ }_{P} t$ and ${ }_{K} t$ and ${ }_{R} t$

We define the mathematical identity.

$$
{ }_{R} t \equiv{ }_{P} t+{ }_{K} t .
$$

Definition: The relationship between ${ }_{P} g$ and ${ }_{o} g$

We define another mathematical identity too.

$$
{ }_{P} g \equiv \frac{{ }_{P} t}{c^{2}} \equiv \frac{{ }_{O} t \times{ }_{O} t}{{ }_{R} t \times c^{2}} \equiv \frac{{ }_{O} t}{{ }_{R} t \times c^{2}} \times{ }_{O} t \equiv \sqrt[2]{1-\frac{v^{2}}{c^{2}}} \times \frac{{ }_{O} t}{c^{2}}=\sqrt[2]{1-\frac{v^{2}}{c^{2}}} \times{ }_{O} g .
$$

Definition: The relationship between ${ }_{R} g$ and ${ }_{W} t$ and ${ }_{R} t$

We define the mathematical identity.

$$
{ }_{K} g \equiv \frac{{ }_{K} t}{c^{2}} \equiv \frac{{ }_{W} t \times{ }_{W} t}{{ }_{R} t \times c^{2}} \equiv \frac{{ }_{W} t}{{ }_{R} t \times c^{2}} \times{ }_{W} t \equiv \frac{v^{2} \times c^{2} \times{ }_{R} g^{2}}{c^{2} \times{ }_{R} g \times c^{2}} \equiv \frac{v^{2} \times{ }_{R} g}{c^{2}} .
$$

Scholium.

In our understanding, ${ }_{k} g$ represents something similar to the gravitational field as associated with the time as determined by an observer which is at rest relative to the relativistic kinetic energy ${ }_{K} E$.

Definition: The relationship between ${ }_{R} g$ and ${ }_{\text {Red }} t$

We define another mathematical identity.

$$
\text { Kred } t=v \times{ }_{R} g .
$$

Definition: The relationship between ${ }_{R} g$ and ${ }_{R e d} t$ and ${ }_{R e d} g$

We define the mathematical identity.

$$
\text { Kred } g \equiv \frac{\text { Kred } t}{c^{2}} \equiv \frac{v \times{ }_{R} g}{c^{2}} .
$$

Definition: The Relationship between ${ }_{w} g$ and ${ }_{w} t$

We define another mathematical identity.

$$
{ }_{w} g=\frac{{ }_{W} t}{c^{2}} .
$$

Equally, it is

$$
{ }_{w} g^{2}=\frac{{ }_{W} t^{2}}{c^{2} \times c^{2}}
$$

Scholium. 
In our understanding, $w g$ represents the gravitational waves.

Definition: The relationship between ${ }_{w} g$ and ${ }_{W} t$

We define the mathematical identity.

$$
{ }_{W} g \equiv \frac{{ }_{W} t}{c^{2}} \equiv \frac{v \times{ }_{R} g \times c}{c^{2}} \equiv \frac{\text { Kred } t \times C}{c^{2}} \equiv{ }_{\text {Kred }} g \times C \equiv \frac{\text { Kred }}{C}=\frac{v}{C} \times{ }_{R} g \text {. }
$$

Definition: The distance ${ }_{o} d$ and the distance ${ }_{R} d$

In general it is distance $=$ speed $\times$ time. The time as such depends on the frame of reference in which it is measured or in other words a moving clock will more slowly. In general, it follows that

$$
{ }_{O} d \equiv c \times{ }_{O} t \equiv c \times{ }_{R} t \times \sqrt[2]{1-\frac{v^{2}}{c^{2}}} \equiv{ }_{R} d \times \sqrt[2]{1-\frac{v^{2}}{c^{2}}}
$$

where ${ }_{o} d$ denotes the distance as measured by a moving observer, ${ }_{o} t$ denotes time as measured by a moving observer, ${ }_{R} d$ denotes the distance as measured by a stationary observer, ${ }_{R} t$ denotes the time as measured by a stationary observer, $v$ denotes the relative velocity between the moving $\mathrm{O}$ and the stationary $\mathrm{R}$ observer and $c$ denotes the speed of light in vacuum.

\subsubsection{Quantum Theory}

\section{Definition: The quantum mechanical energy operator ${ }_{R} H$}

In quantum mechanics, energy is defined in terms of an energy operator which itself is acting on the wavefunction ${ }_{R} \Psi$ of the system. The Hamiltonian, named after Sir William Rowan Hamilton (1805-1865), an Irish mathematician, physicist and astronomer, is a quantum mechanical operator corresponding to the total energy of a quantum mechanical system and usually denoted by $\mathrm{H}$ or by ${ }_{R} H$. By analogy with classical mechanics and special relativity, the Hamiltonian is the sum of operators i.e. is corresponding to the total energy (i.e. potential and kinetic energies) (of all the particles) associated with a quantum mechanical system and can take different forms depending on the situation. The total (relativistic or non-relativistic) energy of a system is transformed into the Hamiltonian which acts as a source of the wavefunction and upon the wavefunction to generate the evolution of the wavefunction in time and space. The Hamiltonian operator is Hermitian. According to the expansion postulate, the wavefunction can be expanded as a series of its eigenfunctions where an eigenfunction belongs to an eigenvalue of the Hamiltonian operator. Consequently, an eigenstate of the operator $\mathrm{H}$ is one in which the energy is perfectly defined. Thus far, an important property of Hermitian operators is that their eigenvalues are real. The total energy operator $\mathrm{H}$ is determined as

$$
{ }_{R} H=H=\hat{H}=i \hbar \frac{\partial}{\partial t} .
$$

For our purposes, the (non-relativistic or relativistic) Hamiltonian is corresponding to the total energy of a (quantum mechanical object or) system. Thus far, it is

$$
{ }_{R} E \equiv{ }_{R} H \equiv H \equiv \hat{H}=i \hbar \frac{\partial}{\partial t}
$$

where ${ }_{R} E$ is identical with the notion "relativistic" energy of a (quantum mechanical) system, $i$ is the imaginary unit, $\hbar$ is the reduced Planck constant, and $H$ is the Hamiltonian operator.

Definition: The quantum mechanical operator of matter ${ }_{R} M$

In quantum mechanics, the Hamiltonian, named after the Irish mathematician Hamilton, is the total energy operator. Thus far we define the quantum mechanical operator of matter ${ }_{R} M$ as

$$
{ }_{R} M \equiv \hat{M} \equiv \frac{{ }_{R} E}{c^{2}} \equiv \frac{H}{c^{2}}=\frac{\hat{H}}{c^{2}}=\frac{i \hbar}{c^{2}} \frac{\partial}{\partial t}
$$

where ${ }_{R} M$ is quantum mechanical operator of matter (and not only of mass), $c$ is the speed of the light in vacuum and $H$ is the Hamiltonian operator.

Scholium.

Matter and energy are equivalent but not identical and thus far not absolutely the same. Einstein himself de- 
fined matter not in relation to time but in relation to gravitational field. Due to Einstein's understanding of the relationship between matter and gravitational field, all but the gravitational field is matter. Consequently, matter as such includes matter in the ordinary sense and the electromagnetic field as well. In other words, there is no third between matter and gravitational field, tertium non datur. Einstein himself wrote:

"Wir unterscheiden im folgenden zwischen 'Gravitationsfeld' und 'Materie', in dem Sinne, daß alles außerdem Gravitationsfeld als 'Materie' bezeichnet wird, also nicht nur die 'Materie' im üblichen Sinne, sondern auch das elektro-magnetische Feld” [13].

Einstein's writing translated into English:

"We make a distinction hereafter between 'gravitational field' and 'matter' in this way, that we denote everything but the gravitational field as 'matter', the word matter therefore includes not only matter in the ordinary sense, but the electromagnetic field as well."

Definition: The eigenvalue $o H$ and the anti eigenvalue $\underline{o} \underline{H}$ of the Hamiltonian operator ${ }_{R} H$

Let us consider i.e. the Hamiltonian ${ }_{R} H$, a physical quantity which characterizes the total energy of a quantum system. Strictly, we should speak in the following discussion not of one quantity, but of a complete set of them. For brevity and simplicity, the values which a given physical quantity like the Hamiltonian can take are called its eigenvalues. The set of these eigenvalues is referred to as the spectrum of eigenvalues of a given quantity. In cases where the eigenvalues of a given physical quantity occupy a continuous range, in such cases we speak of a continuous spectrum of eigenvalues. A given physical quantity can be determined by eigenvalues who form some discrete set. In such cases we speak of a discrete spectrum. We shall suppose for simplicity that the quantities considered here has a discrete spectrum. The case of a continuous spectrum can be discussed later.

The Hamiltonian operator, which plays a central role in quantum mechanics, corresponds to the total energy of a system. An energy eigenstate which does not change by time is called a stationary state. Energy eigenvalues of the Hamiltonian operator are related to the observed values in experimental measurements (i.e. after the "collapse" of the wavefunction). In a single experiment, a measured value ${ }_{O} H$ of the Hamiltonian operator ${ }_{R} H$ is an energy eigenvalue of the Hamiltonian operator. Thus far, let ${ }_{0} H$ denote an energy eigenvalue of the Hamiltonian

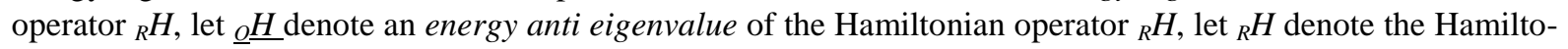
nian operator. In general, we define

$$
{ }_{0} H+\underline{{ }_{0} H} \equiv{ }_{R} H .
$$

Scholium.

Eigenvalues ${ }_{O} H$ of the Hamiltonian operator ${ }_{R} H$ are related to observed values in experimental measurements and thus far determined by the necessities of special theory of relativity. In this context, it is possible that the energy eigenvalue ${ }_{O} H$ is the part of the "relativistic" energy ${ }_{R} E$ which is measured by a co-moving observer O. In other words, there are circumstances where ${ }_{O} E={ }_{O} H$. Under these circumstances, it follows that $\underline{Q} \underline{H}$ is equivalent with $\underline{Q} \underline{H}={ }_{R} E-{ }_{O} E={ }_{\Delta} E$ and quantum theory reduces to simple special theory of relativity. Following Bohm's terminology, $\underline{\underline{H}} \underline{\mathrm{H}}$ can be understood as a kind of a "local hidden" variable. If the energy eigenvalue is measured by a potential observer, it makes sense to denote the same as ${ }_{P} H$. The energy anti eigenvalue of a potential observer, denoted by ${ }_{\underline{P}} \underline{H}$, is then ${ }_{\underline{P}} \underline{H}={ }_{R} H-{ }_{P} H$.

Definition: Eigenfunctions of the Hamiltonian operator ${ }_{R} H$

The Hamiltonian operator, equal to the total energy of a system, is Hermitean. An eigenstate of the operator ${ }_{R} H$ is one in which the energy is perfectly defined and equal to the eigenvalue i.e. ${ }_{i} H$. To each eigenvalue ${ }_{i} H$ of the Hamiltonian operator ${ }_{R} H$ is associated an eigenfunction. A state in which the energy is well defined is a state in which the probabilities remain constant with time and can therefore be called a stationary state.

Definition: The expectation value $E\left({ }_{R} H\right)$ of the Hamiltonian operator ${ }_{R} H$

Let $E\left({ }_{R} H\right)$ denote the expectation value of the Hamiltonian operator ${ }_{R} H$. In general, we define the expectation value of the Hamiltonian operator ${ }_{R} H$ as

$$
E\left({ }_{R} H\right) \equiv{ }_{R} p\left({ }_{R} H\right) \times{ }_{R} H \equiv\left(1-{ }_{R} \Psi\left({ }_{R} X,{ }_{R} t\right) \times{ }_{R} \Psi^{*}\left({ }_{R} X,{ }_{R} t\right)\right) \times{ }_{R} H .
$$

Definition: The complex number ${ }_{R} Y$

Let ${ }_{R} Y$ denote a (complex) number from the standpoint of a stationary observer $R$ of preliminary unknown properties.

Definition: The wave function (of quantum mechanical system) 
Let ${ }_{R} \Psi\left({ }_{R} X,{ }_{R} t\right)$ denote the wavefunction of system from the standpoint of a stationary observer $R$. The wave function represents something like a probability amplitude. When only one spatial dimension ${ }_{R} X$ is relevant we write

$$
{ }_{R} \Psi\left({ }_{R} X,{ }_{R} t\right)
$$

while the modulus squared $\left.\left.\right|_{R} \Psi\left({ }_{R} X\right.$, $\left.{ }_{R} t\right)\right|^{2}$ of the wavefunction gives the probability for finding a quantum mechanical entity at a given point ${ }_{R} X$ in space at the given time ${ }_{R} t$. The wavefunction ${ }_{R} \Psi\left({ }_{R} X,{ }_{R} t\right)$ itself is not an $e i-$ genfunction of an operator. However, every wavefunction (that is not an eigenfunction) can be expressed as a superposition of eigenfunctions of an operator.

\section{Scholium.}

The wavefunction ${ }_{R} \Psi\left({ }_{R} X,{ }_{R} t\right)$ corresponding to the system state can change (out of itself or by a third [i.e. measurement]) into an eigenfunction ${ }_{O} \Psi\left({ }_{O} X\right.$, o $\left.{ }_{o} t\right)$. The change of the wavefunction ${ }_{R} \Psi\left({ }_{R} X,{ }_{R} t\right)$ into an eigenfunction ${ }_{O} \Psi\left({ }_{o} X\right.$, $\left.{ }_{o} t\right)$ of an operator (corresponding to the measured quantity) is called wavefunction collapse. Corresponding to each eigenvalue is an "eigenfunction", corresponding to each anti eigenvalue is an "anti eigenfunction". Only certain eigenvalues with associated eigenfunctions are able to satisfy Schrödinger's equation. The eigenvalue ${ }_{\mathrm{O}} \mathrm{H}$ as measured by a co-moving observer $\mathrm{O}$ is one of the eigenvalues of the quantum mechanical observable ${ }_{R} H$. The eigenvalue (corresponding to some scalar) concept as such is not limited only to energy. Finding a specific function (i.e. eigenfunction) which describes an energy state (i.e. a solution to the Schrodinger equation) is very important. Under conditions where the eigenvalues are discrete, a physical variable is said to be "quantized" and an index $i$ plays the role of a "quantum number" which is characterizing a specific state.

\section{Definition: Born's rule}

The probability of an existing quantum mechanical entity (photon, electron, $\mathrm{X}$-ray, etc.) being somewhere in space is unity. Thus far, the wavefunction must fulfill some certain mathematical requirements, especially the normalization condition. In other words, the integration of the wavefunction over all space leads to a probability of 1 . That is, the wavefunction is normalized. In general, the wavefunction as such represents the probability amplitude for finding a particle ata given point in space at a given time. Due to special theory of relativity, time as measured by a stationary observer can be different from time as determined in the same respect by a moving observer. Thus far, let us define the following.

Let the wavefunction ${ }_{R} \Psi\left({ }_{R} X,{ }_{R} t\right)$ denote the single-valued probability amplitude at $\left.{ }_{R} X,{ }_{R} t\right)$ where ${ }_{R} X$ is position and ${ }_{R} t$ is time i.e. from the standpoint of a stationary observer. Let ${ }_{R} \Psi^{*}\left({ }_{R} X,{ }_{R} t\right)$ denote the complex conjugate of the wavefunction ${ }_{R} \Psi\left({ }_{R} X\right.$, $\left.{ }_{R} t\right)$ i.e. from the standpoint of a stationary observer. Let ${ }_{R} p\left({ }_{R} \Psi\left({ }_{R} X,{ }_{R} t\right)\right)$ denote the probability i.e. from the standpoint of a stationary observer R as associated with the wavefunction ${ }_{R} \Psi\left({ }_{R} X\right.$, $\left.{ }_{R} t\right)$ that a "particle" will be found at $\left({ }_{R} X,{ }_{R} t\right)$.

Let the eigenfunction ${ }_{O} \Psi\left({ }_{o} X\right.$, $\left.{ }_{o} t\right)$ denote the single-valued probability amplitude at ${ }_{o} X$, $\left.{ }_{o} t\right)$ where ${ }_{o} X$ is position and ${ }_{o} t$ is time i.e. from the standpoint of a co-moving observer. Let ${ }_{O} \Psi^{*}\left({ }_{o} X,{ }_{o} t\right)$ denote the complex conjugate of the eigenfunction ${ }_{O} \Psi\left({ }_{o} X\right.$, $\left.{ }_{o} t\right)$ i.e. from the standpoint of a co-moving observer. Let ${ }_{o} p\left({ }_{o} \Psi\left({ }_{o} X\right.\right.$, $\left.\left.{ }_{o} t\right)\right)$ denote the probability i.e. from the standpoint of a co-moving observer $O$ as associated with an eigenfunction that a "particle" will be found at $\left({ }_{o} X,{ }_{o} t\right)$.

In general, due the Born's rule, named after Max Born [14], it is

$$
\left.{ }_{R} p\left({ }_{R} \Psi\left({ }_{R} X,{ }_{R} t\right)\right) \equiv{ }_{R} \Psi\left({ }_{R} X,{ }_{R} t\right) \times{ }_{R} \Psi{ }^{*}{ }_{R} X,{ }_{R} t\right) \equiv\left|{ }_{R} \Psi\left({ }_{R} X,{ }_{R} t\right)\right|^{2}
$$

and

$$
{ }_{o} p\left({ }_{o} \Psi\left({ }_{o} X,{ }_{o} t\right)\right) \equiv{ }_{o} \Psi\left({ }_{o} X,{ }_{o} t\right) \times{ }_{o} \Psi^{*}\left({ }_{o} X,{ }_{o} t\right) \equiv\left|{ }_{o} \Psi\left({ }_{o} X,{ }_{o} t\right)\right|^{2}
$$

where ${ }_{R} X$ is the position as determined by the stationary observer, ${ }_{R} t$ is the time as determined by the stationary observer, the asterix ${ }_{R} \Psi\left({ }_{R} X,{ }_{R} t\right)^{*}$ indicates the complex conjugate of the wavefunction ${ }_{R} \Psi\left({ }_{R} X,{ }_{R} t\right),{ }_{R} p\left({ }_{R} \Psi\left({ }_{R} X,{ }_{R} t\right)\right)$ denotes the probability (mass function or the probability density function or the cumulative distribution function) as associated with the wavefunction. From these definitions follows that

$$
{ }_{R} \Psi\left({ }_{R} X,{ }_{R} t\right) \equiv \frac{1}{{ }_{R} \Psi^{*}\left({ }_{R} X,{ }_{R} t\right)} \times{ }_{R} p\left({ }_{R} \Psi\left({ }_{R} X,{ }_{R} t\right)\right) \equiv \frac{\left|{ }_{R} \Psi\left({ }_{R} X,{ }_{R} t\right)\right|^{2}}{{ }_{R} \Psi^{*}\left({ }_{R} X,{ }_{R} t\right)}
$$

and that 


$$
{ }_{o} \Psi\left({ }_{o} X,{ }_{o} t\right) \equiv \frac{1}{{ }_{O} \Psi^{*}\left({ }_{o} X,{ }_{o} t\right)} \times{ }_{o} p\left({ }_{o} \Psi\left({ }_{o} X,{ }_{o} t\right)\right) \equiv \frac{\left|{ }_{o} \Psi\left({ }_{o} X,{ }_{o} t\right)\right|^{2}}{{ }_{O} \Psi^{*}\left({ }_{o} X,{ }_{o} t\right)} .
$$

Scholium.

The wavefunction satisfies the Schrödinger equation, a first order differential equation in time. Other prefer to use the wavefunction as associated with a random variable instead of the probability as such. Due to the relationship above, this can be done in a contradiction free way.

\section{Definition: The relationship between expansion coefficients and probability}

In general, let ${ }_{R} X$ denote a random variable, an operator, a quantum mechanical observable et cetera, let ${ }_{i} X$ denote an exact numerical (eigen-) value of the random variable ${ }_{R} X$. Let $p\left({ }_{i} X\right)$ denote the probability that in a measurement a system will be found in a state in which the random variable ${ }_{R} X$ has the exact numerical value ${ }_{i} X$. Let $c\left({ }_{i} X\right)$ denote an arbitrary (complex) coefficients. Let $\left.c^{*}{ }_{i} X\right)$ denote arbitrary (complex conjugate) coefficients. Thus, we define

$$
p\left({ }_{i} X\right) \equiv c\left({ }_{i} X\right) \times c^{*}\left({ }_{i} X\right) .
$$

\section{Definition: The expectation value of the wavefunction}

Let $E\left({ }_{R} \Psi\left({ }_{R} X,{ }_{R} t\right)\right)$ denote the expectation value of the wavefunction ${ }_{R} \Psi\left({ }_{R} X,{ }_{R} t\right)$. In general, we define the expectation value of the wave function as

$$
E\left({ }_{R} \Psi\left({ }_{R} X,{ }_{R} t\right)\right) \equiv{ }_{R} p\left({ }_{R} \Psi\left({ }_{R} X,{ }_{R} t\right)\right) \times{ }_{R} \Psi\left({ }_{R} X,{ }_{R} t\right) \equiv{ }_{R} \Psi\left({ }_{R} X,{ }_{R} t\right)^{2} \times{ }_{R} \Psi{ }^{*}\left({ }_{R} X,{ }_{R} t\right) .
$$

\section{Definition: The relationship between the complex number ${ }_{R} Y$ and Born's rule}

Let ${ }_{R} Y$ denote an unknown complex number from the standpoint of a stationary observer $R$. Let the relationship between the unknown complex number ${ }_{R} Y$ and Born's rule be given by

$$
{ }_{R} p\left({ }_{R} \Psi\left({ }_{R} X,{ }_{R} t\right)\right) \equiv{ }_{R} \Psi\left({ }_{R} X,{ }_{R} t\right) \times{ }_{R} \Psi^{*}\left({ }_{R} X,{ }_{R} t\right) \equiv{ }_{R} \Psi\left({ }_{R} X,{ }_{R} t\right) \times{ }_{R} Y .
$$

Scholium.

Due to this definition it is

$$
{ }_{R} Y \equiv{ }_{R} \Psi^{*}\left({ }_{R} X,{ }_{R} t\right) \equiv \frac{{ }_{R} p\left({ }_{R} \Psi\left({ }_{R} X,{ }_{R} t\right)\right)}{{ }_{R} \Psi\left({ }_{R} X,{ }_{R} t\right)} .
$$

\section{Definition: The superposition principle}

The wavefunction ${ }_{R} \Psi\left({ }_{R} X,{ }_{R} t\right)$ itself is not an eigenfunction of an operator. However, every wavefunction can be expressed as a superposition of eigenfunctions of an operator. In general, a set of eigenfunctions of an operator can be used as a basis set. Thus far, any arbitrary wavefunction can be expanded in terms of a superposition of eigenfunctions. In general, assumed that only one spatial dimension is relevant, the wave function can be written briefier as

$$
{ }_{R} \Psi\left({ }_{R} X,{ }_{R} t\right) \equiv \sum_{i=0}^{n}{ }_{i} c \times{ }_{i} \Psi\left({ }_{i} X,{ }_{i} t\right) \equiv{ }_{0} c \times{ }_{0} \Psi\left({ }_{O} X,{ }_{O} t\right)+\sum_{i=1}^{n}{ }_{i} c \times{ }_{i} \Psi\left({ }_{i} X,{ }_{i} t\right)
$$

where ${ }_{i} \Psi\left({ }_{i} X,{ }_{i} t\right)$ denotes the eigenfunction of an corresponding eigenvalue and ${ }_{i} c$ denotes the associated arbitrary complex number. The equation before can be rewritten using Dirac's notation as

$$
\left.\left|{ }_{R} \Psi\right\rangle \equiv \sum_{i=0}^{n}{ }_{i} c \times\left.\right|_{i} \Psi\right\rangle .
$$

The set of all eigenfunctions are called a complete set of states, or a basis. Multiplying with the complex conjugate of the wavefunction $\Psi\left({ }_{R} X,{ }_{R} t\right)^{*}$, we obtain

$$
{ }_{R} p\left({ }_{R} \Psi\left({ }_{R} X,{ }_{R} t\right)\right) \equiv{ }_{R} \Psi\left({ }_{R} X,{ }_{R} t\right) \times{ }_{R} \Psi^{*}\left({ }_{R} X,{ }_{R} t\right) \equiv \sum_{i=0}^{n}{ }_{i} C \times{ }_{i} \Psi\left({ }_{i} X,{ }_{i} t\right) \times{ }_{R} \Psi^{*}\left({ }_{R} X,{ }_{R} t\right)
$$

From this follows that 


$$
\sum_{i=0}^{n}{ }_{i} c \times{ }_{i} \Psi\left({ }_{i} X,{ }_{i} t\right) \times{ }_{i} c^{*} \times{ }_{i} \Psi^{*}\left({ }_{i} X,{ }_{i} t\right) \equiv \sum_{i=0}^{n}{ }_{i} c \times{ }_{i} c^{*} \int \Psi \Psi^{*} \mathrm{~d} X
$$

Scholium.

Eigenfunctions play an important role in quantum physics. Corresponding to each eigenvalue ${ }_{i} E$ is an eigenfunction ${ }_{i} \Psi\left({ }_{i} X,{ }_{i} t\right)$.

\section{Definition: The anti eigenfunction}

Let us now clarify how eigenfunctions and anti eigenfunctions are related to what is observed in experiments. Thus far let us consider the physical quantity ${ }_{R} H$, which characterizes the total energy of a quantum system. For brevity and simplicity we should speak in the following (from the standpoint of the stationary observer $R$ ) not of one quantity, but of a complete set at the same time. Generally speaking, the values which a given physical quantity like the Hamiltonian ${ }_{R} H$ can take are called in quantum mechanics its eigenvalues. The set of these eigenvalues is referred to as the spectrum of eigenvalues of the given physical quantity. In classical mechanics, quantities run through a continuous series of values. Let us suppose for simplicity that the physical quantity ${ }_{R} H$ considered here has a discrete spectrum. The eigenvalues of the physical quantity ${ }_{R} H$ are denoted by ${ }_{i} H$, where the suffix $i$ takes the values $0,1,2,3, \cdots$. The associated wave function of the system, in the state where the physical quantity ${ }_{R} H$ has the value ${ }_{i} H$ is denoted by ${ }_{i} \Psi\left({ }_{i} X,{ }_{i} t\right)$. The wave functions ${ }_{i} \Psi\left({ }_{i} X,{ }_{i} t\right)$ are called the eigenfunctions of the given physical quantity ${ }_{R} H$. In accordance with the principle of superposition, the eigenfunctions form a complete set. Thus far, any wave function can be written as a linear combination of eigenfunctions. Let ${ }_{O} \Psi\left({ }_{o} X, o_{o} t\right)$ denote the eigenfunction (i.e. as determined by the moving observer $O$ ). Let ${ }_{0} \Psi\left({ }_{0} X\right.$, $\left.{ }_{0} t\right)$ denote the corresponding anti eigenfunction. In this respect, let $\Psi\left({ }_{R} X,{ }_{R} t\right)$ denote the wavefunction of a system from the standpoint of a stationary observer $R$. The wave function can be expanded in terms of the eigenfunctions and represented in the form of a series as

$$
{ }_{R} \Psi\left({ }_{R} X,{ }_{R} t\right) \equiv \sum_{i=O}^{n=\ldots}{ }_{i} c \times{ }_{i} \Psi\left({ }_{i} X,{ }_{i} t\right) \equiv{ }_{O} C \times{ }_{O} \Psi\left({ }_{O} X,{ }_{O} t\right)+\sum_{i=1}^{n=\ldots \ldots}{ }_{i} c \times{ }_{i} \Psi\left({ }_{i} X,{ }_{i} t\right)
$$

where the summation extends over all $n$, and the ${ }_{i} c$ are some arbitrary complex numbers. In general, we define an anti-eigenfunction $\underline{{ }_{O} \Psi}\left(\underline{{ }_{0} X},{ }_{0} t\right)$ of the eigenfunction ${ }_{O} \Psi\left({ }_{o} X,{ }_{o} t\right)$ as

$$
\underline{{ }_{O} C \times} \underline{O} \Psi\left(\underline{{ }_{0} X}, \underline{{ }_{O} t}\right)={ }_{R} \Psi\left({ }_{R} X,{ }_{R} t\right)-{ }_{O} C \times{ }_{O} \Psi\left({ }_{O} X,{ }_{O} t\right)
$$

or as

$$
\underline{{ }_{O} C} \times \underline{O} \underline{O}\left(\underline{{ }_{O} X}, \underline{{ }_{O}} t\right) \equiv \sum_{i=1}^{n}{ }_{i} C \times{ }_{i} \Psi\left({ }_{i} X,{ }_{i} t\right) \equiv{ }_{R} \Psi\left({ }_{R} X,{ }_{R} t\right)-{ }_{O} C \times{ }_{O} \Psi\left({ }_{O} X,{ }_{O} t\right) .
$$

The anti-eigenfunction of the observer $\mathrm{k}$ is defined as

$$
\underline{{ }_{k}} C \times \underline{{ }_{k}} \Psi\left(\underline{{ }_{k} X}, \underline{{ }_{k}} t\right) \equiv{ }_{R} \Psi\left({ }_{R} X,{ }_{R} t\right)-{ }_{k} C \times{ }_{k} \Psi\left({ }_{k} X,{ }_{k} t\right) .
$$

Each coefficient ${ }_{i} c$ in the expansion determines the probability of the corresponding physical quantity. The sum of the probabilities of all possible values must be equal to unity. In other words, at the end the relation

$$
\left.\left.\sum_{i=0}^{n=\ldots}\right|_{i} c\right|^{2} \equiv 1
$$

must hold.

\section{Scholium.}

The above definition assures the compatibility of quantum theory with relativity theory and does not exclude the possibility that an eigenfunction can be determined by a potential observer P (i.e. from the standpoint of potential energy). In this case we would obtain

$$
\underline{{ }_{P} C} \times \underline{{ }_{P} \Psi}\left(\underline{{ }_{P} X}, \underline{{ }_{P}} t\right) \equiv{ }_{R} \Psi\left({ }_{R} X,{ }_{R} t\right)-{ }_{P} C \times{ }_{P} \Psi\left({ }_{P} X,{ }_{P} t\right)
$$

where ${ }_{P} \Psi\left({ }_{P} X,{ }_{P} t\right)$ denotes the eigenfunction as determined by the observer $P$ and ${ }_{P} \Psi\left(\underline{{ }_{P}} X,{ }_{P} t\right)$ denotes the cor- 
responding anti-eigenfunction.

\section{Definition: The complex number ${ }_{R} S$}

Let ${ }_{R} S$ denote something, i.e. a (complex) number from the standpoint of a stationary observer $R$. Let $o S$ denote a (complex) number of the same system in the same respect from the standpoint of a co-moving observer. In general, we define

$$
{ }_{R} S \equiv{ }_{R} \Psi\left({ }_{R} X,{ }_{R} t\right)+{ }_{R} H \equiv{ }_{R} \Psi\left({ }_{R} X,{ }_{R} t\right)+i \hbar \frac{\partial}{\partial_{R} t}
$$

where ${ }_{R} H$ denotes the Hamiltonian from the standpoint of a stationary observer $R,{ }_{R} \Psi(t)$ denote the wavefunction of the system from the standpoint of a stationary observer $R$, where $i$ is the imaginary unit, $\hbar$ is the Planck constant divided by $2 \times \pi, \frac{\partial}{\partial t}$ indicates a partial derivative with respect to time $t$.

Perhaps there is no need for us to point out that there are other approaches to the notion energy. The topic of this definition is not—at least directly—energy as such; rather, it is the definition of the relationship between energy and time. In general, it is

$$
{ }_{R} E+{ }_{R} t={ }_{R} S .
$$

\section{Definition: The complex conjugate ${ }_{R} S^{*}$ of the complex number ${ }_{R} S$}

The complex conjugate of ${ }_{R} S^{*}$ of the complex number ${ }_{R} S$ from the standpoint of a stationary observer $R$ is defined as

$$
{ }_{R} S^{*} \equiv{ }_{R} \Psi(t)-i \hbar \frac{\partial}{\partial t}
$$

\section{Scholium.}

In our understanding of the relationship between energy and time everything but time is energy. More precisely, there is no third between energy and time. Clearly, ${ }_{R} S$ is a kind of a complex number. Thus far, the straight forward question is, are there circumstances where ${ }_{R} S \times{ }_{R} S^{*}=i^{2}=1$ ?

Definition: The relationship between the complex number ${ }_{R} Y$ and the complex number ${ }_{R} S$

Let ${ }_{R} Y$ denote an unknown complex number from the standpoint of a stationary observer $R$. Let the relationship between the unknown complex number ${ }_{R} Y$ and the unknown complex number ${ }_{R} S$ be given by

$$
{ }_{R} S \times{ }_{R} Y \equiv{ }_{R} \Psi\left({ }_{R} X,{ }_{R} t\right) \times{ }_{R} Y+{ }_{R} H \times{ }_{R} Y \equiv{ }_{R} \Psi\left({ }_{R} X,{ }_{R} t\right) \times{ }_{R} Y+i \hbar \frac{\partial}{\partial_{R} t} \times{ }_{R} Y \equiv 1 .
$$

Scholium.

Due to this definition it is

$$
{ }_{R} Y \equiv \frac{1}{{ }_{R} S} .
$$

Definition: The complex number ${ }_{o} S$ and $\underline{o} \underline{S}$

Let ${ }_{O} S$ denote something, i.e. a (complex) number from the standpoint of an eigenvalue and eigenfunction (i.e. a co-moving observer O). Let $\underline{\underline{S}} \underline{S}$ denote an anti (complex) number of the same system. In general, we define

$$
{ }_{o} S \equiv{ }_{O} H+{ }_{O} C \times{ }_{O} \Psi\left({ }_{o} X,{ }_{o} t\right)
$$

and

$$
\underline{{ }_{O} S} \equiv \underline{{ }_{O} H}+\underline{{ }_{O} C \times{ }_{O} \Psi\left({ }_{O} X,{ }_{O} t\right)}
$$

and

$$
{ }_{R} S \equiv{ }_{O} S+{ }_{O} S={ }_{R} \Psi\left({ }_{R} X,{ }_{R} t\right)+{ }_{R} H .
$$

Scholium. 
The following picture may illustrate the definitions above.

\begin{tabular}{|c|c|c|c|c|}
\hline \multirow{2}{*}{\multicolumn{2}{|c|}{ Fig. }} & \multicolumn{2}{|c|}{ “Curvature” } & \\
\hline & & yes & no & \\
\hline \multirow{2}{*}{ “Momentum” } & yes & ${ }_{0} H$ & $\underline{{ }_{0} H}$ & ${ }_{R} H$ \\
\hline & no & ${ }_{0} C \times{ }_{0} \Psi\left({ }_{0} X,{ }_{0} t\right)$ & $\underline{{ }_{0} C \times{ }_{0} \Psi\left({ }_{0} X,{ }_{o} t\right)}$ & ${ }_{R} \Psi\left({ }_{R} X,{ }_{R} t\right)$ \\
\hline & & ${ }_{0} S$ & ${ }_{0} S$ & ${ }_{R} S$ \\
\hline
\end{tabular}

The four notions $\left({ }_{o} H, \underline{O} \underline{H},{ }_{O} \Psi, \underline{O} \underline{\Psi}\right)$ can denote the four basic fields of nature too. From the above definitions follows that

$$
{ }_{R} S-{ }_{R} \Psi\left({ }_{R} X,{ }_{R} t\right) \equiv{ }_{O} S+{ }_{O} S-{ }_{R} \Psi\left({ }_{R} X,{ }_{R} t\right)=+{ }_{R} H .
$$

Let us assume, just for the sake of the argument, that there are conditions where

$$
\Lambda \equiv \underline{O} S-{ }_{R} \Psi\left({ }_{R} X,{ }_{R} t\right)
$$

where $\Lambda$ denotes Einstein's cosmological constant. Under these circumstances, we obtain

$$
{ }_{O} S+\Lambda=+{ }_{R} H \text {. }
$$

Multiplying by Einstein's metric tensor $g_{\mu v}$ we obtain

$$
{ }_{O} S \times g_{\mu v}+\Lambda \times g_{\mu v}={ }_{R} H \times g_{\mu v} .
$$

Under conditions where $\underline{T}_{\mu v}=\left(8 \times \pi \times \gamma / c^{4}\right) \times T_{\mu \nu}-{ }_{R} H \times g_{\mu v}$ it should be that is $\underline{G}_{\mu v}=G_{\mu \nu}-{ }_{o} S \times g_{\mu v}$. "Historically the term containing the "cosmological constant" was introduced into the field equations in order to enable us to account theoretically for the existence of a finite mean density in a static universe. It now appears that in the dynamical case this end can be reached without the introduction of $\Lambda$ " [15].

\section{Definition: The complex number ${ }_{R} U$}

Let ${ }_{R} U$ denote a complex number from the standpoint of a stationary observer. Let ${ }_{O} U$ denote a complex number of the same system in the same respect from the standpoint of a co-moving observer. Let ${ }_{R} S$ denote a complex number from the standpoint of a stationary observer. Let $o S$ denote a complex number of the same system in the same respect from the standpoint of a co-moving observer. In general, we define

$$
{ }_{R} U \equiv \frac{{ }_{R} S}{c^{2}} \equiv \frac{{ }_{R} \Psi(t)}{c^{2}}+\frac{{ }_{R} H}{c^{2}} \equiv \frac{{ }_{R} \Psi(t)}{c^{2}}+\frac{i \hbar}{c^{2}} \frac{\partial}{\partial t} .
$$

The complex conjugate of ${ }_{R} U^{*}$ of the complex number ${ }_{R} U$ from the standpoint of a stationary observer $R$ is defined as

$$
{ }_{R} U^{*} \equiv \frac{{ }_{R} S^{*}}{C^{2}} .
$$

Definition: The quantum mechanical wave function of the gravitational field ${ }_{R} G$

Let the wavefunction of the gravitational field (from the standpoint of a stationary observer $R$ ) describe the gravitational field completely. In general, let ${ }_{R} G$ denote the wavefunction of the gravitational field from the standpoint of a stationary observer $\mathrm{R}$, let ${ }_{R} G^{*}$ denote the complex conjugate of the wavefunction of the gravitational field from the standpoint of a stationary observer $\mathrm{R}$, let ${ }_{o} G$ denote the wavefunction of the gravitational field from the standpoint of a co-moving observer $\mathrm{O}$, let ${ }_{o} G^{*}$ denote the complex conjugate of the wavefunction of the gravitational field from the standpoint of a co-moving observer O. Let ${ }_{R} M$ denote matter or the quantum mechanical operator of matter (as defined above) from the standpoint of a stationary observer R. In general, we define

$$
{ }_{R} G \equiv{ }_{R} U-{ }_{R} M
$$


Definition: The relationship between matter and gravitational field in general

In general, the relationship between matter and gravitational field is given by

$$
{ }_{R} U \equiv \frac{{ }_{R} S}{C^{2}} \equiv{ }_{R} U+0 \equiv{ }_{R} U-{ }_{R} M+{ }_{R} M \equiv{ }_{R} G+{ }_{R} M .
$$

\section{Scholium.}

There is no third between gravitational field and matter, a third is not given (tertium non datur). Aristotle's (384-322) B.C. principium exclusitertii seumedii inter duo contradictoria follows from this relationship. Still, there is a close relationship between matter i.e. energy and the gravitational field, both are related to each other in a certain manner. Especially, Einstein himself provided some very important views on the relationship between matter and gravitational field.

"Das G-Feld ist restlos durch die Massen der Körper bestimmt” [16].

Translated into English:

"The G-field [gravitational field, author] is completely determined by the masses of the bodies."

Einstein, while investigating the nature of the relationship between matter and the gravitational field, writes:

"Da Masse und Energie nach den Ergebnissen der speziellen Relativitätstheorie das Gleiche sind und die Energie formal durch den symmetrischen Energietensor $\left(T_{\mu v}\right)$ beschrieben wird, so besagt dies, daß das G-Feld durch den Energietensor der Materie bedingt und bestimmt sei” [16].

Einstein's view translated into English:

"According to the results of the special theory of relativity mass and energy are the same and energy itself is described by the symmetric energy tensor $\left(T_{\mu v}\right)$ so this indicates that the G-field [gravitational field, author] is conditioned and determined by the energy tensor of matter"

Gravitational field and matter are not only determined by each other. Both are related to each other in a very subtle way.

"Wir unterscheiden im folgenden zwischen 'Gravitationsfeld' und 'Materie', in dem Sinne, daß alles außer dem Gravitationsfeld als 'Materie' bezeichnet wird, also nicht nur die 'Materie' im üblichen Sinne, sondern auch das elektro-magnetische Feld" [13].

Einstein's position translated into English:

"We make a distinction hereafter between the 'gravitational field' and 'matter' in this way, that we denote everything but the gravitational field as 'matter', the word matter therefore includes not only matter in the ordinary sense, but the electromagnetic field as well."

We see from the above that Einstein himself defined the relationship between matter (i.e. not only mass) and gravitational field ex negativo. More precisely, due to Einstein everything but the gravitational field is matter. Consequently, matter as such includes matter in the ordinary sense and the electromagnetic field as well. In other words, there is no third between matter and gravitational field. For the sake of illustration of the principles involved, let us consider Table 1.

This approach to the relationship between matter and gravitational field is backgrounded by the de Broglie hypothesis too. Louis de Broglie worked on Compton's momentum phenomena. In 1924, as part of his PhD thesis, de Broglie hypothesized that all matter (i.e. electrons) can exhibit wave-like behaviour. Louis de Broglie was awarded the Nobel Prize in Physics 1929 for the discovery of the wave nature of electrons (i.e. matter waves). These matter waves, a central part of today's theory of quantum mechanics, were first experimentally confirmed to occur in the Davisson-Germer experiment [17] for electrons.

\section{Definition: The Schrödinger equation}

The Schrödinger equation for any system, no matter whether relativistic or not, no matter how complicated, has the form

$$
{ }_{R} H \times{ }_{R} \Psi\left({ }_{R} X,{ }_{R} t\right)=i \hbar \frac{\partial}{\partial t}{ }_{R} \Psi\left({ }_{R} X,{ }_{R} t\right),
$$

where $i$ is the imaginary unit, $\hbar=\frac{h}{2 \times \pi}$ is Planck's constant divided by $2 \times \pi$, the symbol $\frac{\partial}{\partial t}$ indicates a partial derivative with respect to time ${ }_{R} t,{ }_{R} \Psi\left({ }_{R} X,{ }_{R} t\right)$ is the wave function of the quantum system, and ${ }_{R} H$ is the Hamiltonian operator. 
Table 1. The relationship between matter and gravitational field..

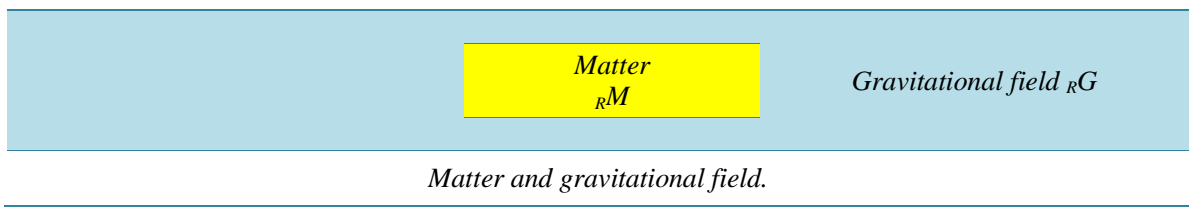

\subsection{Axioms}

\section{Axiom I. (Lex identitatis).}

The following theory is based on the following axiom:

$$
+1=+1 .
$$

Scholium.

Why does it make sense to define such an axiom and to take all transformations and proofs from the same? An axiom should be so evident or well-established, that it is or can be accepted without any controversy or question by the scientific community. Thus far, such an axiom can be used as a premise or as a starting point for further theorems, arguments and reasoning. As we will see, a lot of proofs in this paper are repeated to demonstrate the strategic potential of axiom I. All Newtonian axioms can be derived from the axiom I, Einstein's field equation can be derived from the axiom I. Consequently, axiom I has the potential to serve as the common ground for the unification of quantum and relativity theory into a unique mathematical framework.

\section{Results}

\subsection{Theorem: Newton's First Axiom Can Be Derived from Axiom I}

Claim. (Theorem. Proposition. Statement.)

In general, Newton first law can be derived from axiom I as

$$
\sum_{t=0}^{n} \vec{t} \vec{F}=0 .
$$

Direct proof.

In general, axiom I is determined as

$$
+1=+1 .
$$

Multiplying this equation by 0 , we obtain

$$
0 \times 1=0 \times 1 .
$$

In general it is

$$
0=0
$$

As stated before, Newton's first law first law demands that the net force (a vector sum of all forces acting on something) is zero. We obtain

$$
\sum_{t=0}^{n} \vec{t} \vec{F}=0
$$

Quod erat demonstrandum.

\subsection{Theorem: Newton's Second Axiom Can Be Derived from Axiom I}

Claim. (Theorem. Proposition. Statement.)

In general, Newton second law can be derived from axiom I as

$$
\overrightarrow{{ }_{o} F}={ }_{o} m \times \overrightarrow{{ }_{o} a} \text {. }
$$




\section{Direct proof.}

In general, axiom I is determined as

$$
+1=+1 \text {. }
$$

Multiplying this equation by ${ }_{O} F$, we obtain

$$
\overrightarrow{{ }_{O} F} \times 1=\overrightarrow{{ }_{O} F} \times 1 .
$$

In general it is ${ }_{O} F={ }_{O} m \times{ }_{O} a$ and we obtain

$$
\overrightarrow{{ }_{O} F}={ }_{o} m \times \overrightarrow{{ }_{o} a} .
$$

\section{Quod erat demonstrandum.}

\subsection{Theorem: Newton's Third Axiom Can Be Derived from Axiom I}

Claim. (Theorem. Proposition. Statement.)

In general, Newton third law is defined as

$$
\overrightarrow{{ }_{O} F}=-\overline{{ }_{R} F} \text {. }
$$

Direct proof.

In general, axiom I is determined as

$$
+1=+1 \text {. }
$$

Multiplying this equation by ${ }_{O} F$, we obtain

$$
\overrightarrow{{ }_{o} F}=\overrightarrow{{ }_{o} F} .
$$

Adding ${ }_{R} F$, we obtain

$$
\overrightarrow{{ }_{O} F}+\overrightarrow{{ }_{R} F}=\overrightarrow{{ }_{O} F}+\overrightarrow{{ }_{R} F}=\overrightarrow{{ }_{R} C} \text {. }
$$

Newton's laws of motion are valid in inertial reference frames where the net force is equal to zero. We obtain

$$
\overrightarrow{{ }_{O} F}+\overrightarrow{{ }_{R} F}=\overrightarrow{{ }_{O} F}+\overrightarrow{{ }_{R} F}=\overrightarrow{{ }_{R} C}=0 .
$$

At the end, Newton's third law follows as

$$
\overrightarrow{{ }_{O} F}=-\overrightarrow{{ }_{R} F} \text {. }
$$

\section{Quod erat demonstrandum.}

\section{Scholium.}

In particular, an axiom serves as a starting point from which other theorems, laws, equations, statements et cetera are logically derived and should be something which is so well-established or evident that it can be accepted without question or controversy or demonstration. However, an axiom in one theory or system may be only one theorem in another theory or system, and vice versa. This theory is based on the axiom $+1=+1$. From this axiom, Newton's axioms can be derived without any contradiction. Consequently, in this theory, Newton's axioms are only theorems. The axiom $+1=+1$ is very general and of use outside of physics too. Axiom I can be the foundation for an axiomatic formulations of quantum mechanics.

\subsection{Theorem: Einstein's Field Equations}

Einstein's field equations can be derived from axiom I.

Claim. (Theorem. Proposition. Statement.)

In general, Einstein's field equations are derived as

$$
G_{\mu v}+\left(\Lambda \times g_{\mu v}\right)=\left(\frac{4 \times 2 \times \pi \times \gamma}{c^{4}} \times T_{\mu v}\right) .
$$




\section{Direct proof.}

In general, axiom I is determined as

$$
+1=+1 .
$$

Multiplying this equation by the stress-energy tensor of general relativity $\left((4 \times 2 \times \pi \times \gamma) /\left(c^{4}\right)\right) \times T_{\mu \nu}$, it is

$$
+1 \times\left(\frac{4 \times 2 \times \pi \times \gamma}{c^{4}} \times T_{\mu v}\right)=+1 \times\left(\frac{4 \times 2 \times \pi \times \gamma}{c^{4}} \times T_{\mu v}\right)
$$

where $\gamma$ is Newton's gravitational "constant" [18], $c$ is the speed of light in vacuum and $\pi$, sometimes referred to as “Archimedes' constant”, is the ratio of a circle's circumference to its diameter. Due to Einstein's general relativity, Equation (95) is equivalent with

$$
R_{\mu v}-\left(\frac{R}{2} \times g_{\mu v}\right)+\left(\Lambda \times g_{\mu v}\right)=\left(\frac{4 \times 2 \times \pi \times \gamma}{c^{4}} \times T_{\mu v}\right) .
$$

$R_{\mu v}$ is the Ricci curvature tensor, $R$ is the scalar curvature, $g_{\mu v}$ is the metric tensor, $\Lambda$ is the cosmological constant and $T_{\mu v}$ is the stress-energy tensor. By defining the Einstein tensor as $G_{\mu v}=R_{\mu v}-(R / 2) \times g_{\mu v}$, it is possible to write the Einstein field equations in a more compact as

$$
G_{\mu v}+\left(\Lambda \times g_{\mu v}\right)=\left(\frac{4 \times 2 \times \pi \times \gamma}{c^{4}} \times T_{\mu v}\right) .
$$

\section{Quod erat demonstrandum.}

\subsection{Theorem: The Normalized Relativistic Energy-Momentum Relation}

Claim. (Theorem. Proposition. Statement.)

In general, it is

$$
\frac{{ }_{O} E^{2}}{{ }_{R} E^{2}}+\frac{v^{2}}{c^{2}}=1
$$

Direct proof.

In general, axiom I is determined as

$$
+1=+1 \text {. }
$$

Multiplying this equation by "rest" mass ${ }_{O} m$, it is

$$
{ }_{o} m \times 1={ }_{o} m \times 1 .
$$

Due to special relativity, we rearrange this equation to

$$
{ }_{O} m={ }_{R} m \times \sqrt[2]{1-\frac{v^{2}}{c^{2}}} .
$$

Multiplying by the speed of the light $c$ square, we obtain

$$
{ }_{O} m \times c^{2}={ }_{R} m \times c^{2} \times \sqrt[2]{\left(1-\frac{v^{2}}{c^{2}}\right)} .
$$

This equation is equivalent with

$$
{ }_{O} E={ }_{R} E \times \sqrt[2]{\left(1-\frac{v^{2}}{c^{2}}\right)}
$$

Rearranging this equation, we obtain 


$$
\frac{{ }_{O} E}{{ }_{R} E}=\sqrt[2]{\left(1-\frac{v^{2}}{c^{2}}\right)}
$$

A further mathematical manipulation of the equation before leads to

$$
\frac{{ }_{O} E^{2}}{{ }_{R} E^{2}}=\left(1-\frac{v^{2}}{c^{2}}\right) \text {. }
$$

At the end, we obtain the relationship

$$
\frac{{ }_{O} E^{2}}{{ }_{R} E^{2}}+\frac{v^{2}}{c^{2}}=1 .
$$

Quod erat demonstrandum.

\subsection{Theorem: The Normalized Relativistic Time Dilation Relation}

Claim. (Theorem. Proposition. Statement.)

In general, it is

$$
\frac{{ }_{O} t^{2}}{{ }_{R} t^{2}}+\frac{v^{2}}{c^{2}}=1 \text {. }
$$

Direct proof.

In general, axiom I is determined as

$$
+1=+1 \text {. }
$$

Multiplying this equation by time ${ }_{o} t$ as measured by a co-moving observer, it is

$$
{ }_{o} t \times 1={ }_{o} t \times 1 \text {. }
$$

Due to special relativity, we rearrange this equation as

$$
{ }_{O} t={ }_{R} t \times \sqrt[2]{1-\frac{v^{2}}{c^{2}}} .
$$

This equation is equivalent with

$$
\frac{{ }_{O} t}{{ }_{R} t}=\sqrt[2]{\left(1-\frac{v^{2}}{c^{2}}\right)}
$$

A further mathematical manipulation of the equation before leads to

$$
\frac{{ }_{o} t^{2}}{{ }_{R} t^{2}}=\left(1-\frac{v^{2}}{c^{2}}\right) \text {. }
$$

At the end, we obtain the relationship

$$
\frac{{ }_{o} t^{2}}{{ }_{R} t^{2}}+\frac{v^{2}}{c^{2}}=1 .
$$

\section{Quod erat demonstrandum.}

\subsection{Theorem: The First Basic Law of Special Relativity}

Special relativity implies basic physical law of far reaching consequences.

Claim. (Theorem. Proposition. Statement.) 
Under conditions of special relativity, it is

$$
{ }_{o} E \times{ }_{R} t={ }_{O} t \times{ }_{R} E .
$$

Direct proof.

In general, axiom I is determined as

$$
+1=+1 \text {. }
$$

Due to the theorem before, it is $\left({ }_{O} E /{ }_{R} E+v^{2} / C^{2}=1\right)$. We obtain

$$
\frac{{ }_{O} E}{{ }_{R} E}+\frac{v^{2}}{c^{2}}=1 .
$$

Due to another theorem before, it is $\left({ }_{o} t / R_{R} t+v^{2} / c^{2}=1\right)$. We obtain

$$
\frac{{ }_{O} E}{{ }_{R} E}+\frac{v^{2}}{c^{2}}=\frac{{ }_{O} t}{{ }_{R} t}+\frac{v^{2}}{c^{2}} \text {. }
$$

After subtraction of $\left(v^{2} / c^{2}\right)$, it follows that

$$
\frac{{ }_{O} E}{{ }_{R} E}=\frac{{ }_{0} t}{{ }_{R} t} .
$$

Multiplying this equation by the term $\left({ }_{R} E \times{ }_{R} t\right)$, it is

$$
\frac{{ }_{O} E \times{ }_{R} E \times{ }_{R} t}{{ }_{R} E}=\frac{{ }_{O} t \times{ }_{R} E \times{ }_{R} t}{{ }_{R} t} .
$$

Simplifying this equation, we obtain

$$
{ }_{O} E \times{ }_{R} t={ }_{O} t \times{ }_{R} E .
$$

\section{Quod erat demonstrandum.}

\subsection{Theorem: The Normalization of the Relationship between $o E$ and ${ }_{\Delta} E$}

Claim. (Theorem. Proposition. Statement.)

In general, we must accept that

$$
\frac{{ }_{O} E}{{ }_{R} E}+\frac{{ }_{\Delta} E}{{ }_{R} E}=1 .
$$

Direct proof.

In general, axiom I is determined as

$$
+1=+1
$$

Multiplying this equation by ${ }_{R} E$, we obtain

$$
{ }_{R} E={ }_{R} E .
$$

Due to our definition ${ }_{R} E={ }_{O} E+{ }_{Q} \underline{E}$ or ${ }_{R} E={ }_{O} E+{ }_{\Delta} E$ it is $\underline{Q} \underline{E}={ }_{\Delta} E$ and we obtain

$$
{ }_{O} E+{ }_{\Delta} E={ }_{R} E \text {. }
$$

Dividing this equation by ${ }_{R} E$ it is

$$
\frac{{ }_{O} E}{{ }_{R} E}+\frac{{ }_{\Delta} E}{{ }_{R} E}=1
$$

Quod erat demonstrandum. 


\subsection{Theorem: The Normalization of the Relationship between ${ }_{0} t$ and ${ }_{\Delta} t$}

Claim. (Theorem. Proposition. Statement.)

In general, it is

$$
\frac{{ }_{O} t}{{ }_{R} t}+\frac{{ }_{\Delta} t}{{ }_{R} t}=1
$$

Direct proof.

In general, axiom I is determined as

$$
+1=+1
$$

Multiplying this equation by ${ }_{R} t$, we obtain

$$
{ }_{R} t={ }_{R} t
$$

Our definition was ${ }_{R} t={ }_{O} t+{ }_{\Delta} t$. The equation before changes to

$$
{ }_{O} t+{ }_{\Delta} t={ }_{R} t .
$$

Dividing this equation by ${ }_{R} t$ it is

$$
\frac{{ }_{O} t}{{ }_{R} t}+\frac{{ }_{\Delta} t}{{ }_{R} t}=1
$$

\section{Quod erat demonstrandum.}

\subsection{Theorem: The Second Basic Law of Special Relativity}

Special relativity implies another basic physical law of no less reaching consequences.

Claim. (Theorem. Proposition. Statement.)

Under conditions of special relativity, it is

$$
{ }_{\Delta} E \times{ }_{R} t={ }_{\Delta} t \times{ }_{R} E .
$$

Direct proof.

In general, axiom I is determined as

$$
+1=+1 .
$$

Due to the theorem before, it is $\left({ }_{O} E /{ }_{R} E+{ }_{\Delta} E /{ }_{R} E=1\right)$. We obtain

$$
\frac{{ }_{O} E}{{ }_{R} E}+\frac{{ }_{\Delta} E}{{ }_{R} E}=1 \text {. }
$$

Due to another theorem before, it is $\left({ }_{o} t /{ }_{R} t+{ }_{\Delta} t /{ }_{R} t=1\right)$. We obtain

$$
\frac{{ }_{O} E}{{ }_{R} E}+\frac{{ }_{\Delta} E}{{ }_{R} E}=\frac{{ }_{O} t}{{ }_{R} t}+\frac{{ }_{\Delta} t}{{ }_{R} t} .
$$

After multiplication with $\left({ }_{R} E \times{ }_{R} t\right)$, it follows that

$$
\frac{{ }_{O} E \times{ }_{R} E \times{ }_{R} t}{{ }_{R} E}+\frac{{ }_{\Delta} E \times{ }_{R} E \times{ }_{R} t}{{ }_{R} E}=\frac{{ }_{O} t \times{ }_{R} E \times{ }_{R} t}{{ }_{R} t}+\frac{{ }_{\Delta} t \times{ }_{R} E \times{ }_{R} t}{{ }_{R} t} .
$$

Rearranging equation, it is

$$
{ }_{O} E \times{ }_{R} t+{ }_{\Delta} E \times{ }_{R} t={ }_{O} t \times{ }_{R} E+{ }_{\Delta} t \times{ }_{R} E .
$$

According to the first basic law of special relativity, it is ${ }_{O} E \times{ }_{R} t={ }_{R} E \times{ }_{O} t$. Based on this insight, we rearrange the equation before and do obtain 


$$
{ }_{O} E \times{ }_{R} t+{ }_{\Delta} E \times{ }_{R} t={ }_{O} E \times{ }_{R} t+{ }_{\Delta} t \times{ }_{R} E
$$

or at the end similar the law of the lever as provided and proven by Archimedes ( 287 BC - 212 BC)

$$
{ }_{\Delta} E \times{ }_{R} t={ }_{\Delta} t \times{ }_{R} E .
$$

\section{Quod erat demonstrandum.}

\subsection{Theorem: The Relationship between the (Complex) Number ${ }_{R} S$ and the Unknown Complex Number ${ }_{R} Y$}

Claim. (Theorem. Proposition. Statement.)

In general, it is

$$
{ }_{R} Y \equiv \frac{1}{{ }_{R} S}
$$

Direct proof.

In general, axiom I is determined as

$$
+1=+1 \text {. }
$$

Multiplying this equation by ${ }_{R} S \times{ }_{R} Y$, we obtain

$$
{ }_{R} S \times{ }_{R} Y \times 1 \equiv{ }_{R} S \times{ }_{R} Y \times 1
$$

Due to our above definition, it is ${ }_{R} S \times{ }_{R} Y=1$. Consequently, Equation (141) changes too

$$
{ }_{R} S \times{ }_{R} Y \equiv 1 .
$$

We obtain

$$
{ }_{R} Y \equiv \frac{1}{{ }_{R} S}
$$

\section{Quod erat demonstrandum.}

3.12. Theorem: The Relationship between the Complex Conjugate ${ }_{R} \Psi^{*}\left({ }_{R} X,{ }_{R} t\right)$ of the Wave Function ${ }_{R} \Psi\left({ }_{R} X,{ }_{R} t\right)$ and the Unknown (Complex) Number ${ }_{R} Y$

Claim. (Theorem. Proposition. Statement.)

In general, it is

$$
{ }_{R} Y \equiv{ }_{R} \Psi^{*}\left({ }_{R} X,{ }_{R} t\right) .
$$

\section{Direct proof.}

In general, axiom I is determined as

$$
+1=+1 \text {. }
$$

Multiplying this equation by ${ }_{R} \Psi\left({ }_{R} X,{ }_{R} t\right) \times{ }_{R} \Psi^{*}\left({ }_{R} X,{ }_{R} t\right)$, we obtain

$$
{ }_{R} \Psi\left({ }_{R} X,{ }_{R} t\right) \times{ }_{R} \Psi^{*}\left({ }_{R} X,{ }_{R} t\right) \times 1 \equiv{ }_{R} \Psi\left({ }_{R} X,{ }_{R} t\right) \times{ }_{R} \Psi^{*}\left({ }_{R} X,{ }_{R} t\right) \times 1 .
$$

Due to our above definition, it is ${ }_{R} \Psi\left({ }_{R} X,{ }_{R} t\right) \times{ }_{R} Y={ }_{R} \Psi\left({ }_{R} X,{ }_{R} t\right) \times{ }_{R} \Psi^{*}\left({ }_{R} X,{ }_{R} t\right)$. Consequently, Equation (146) changes too

$$
{ }_{R} \Psi\left({ }_{R} X,{ }_{R} t\right) \times{ }_{R} Y \equiv{ }_{R} \Psi\left({ }_{R} X,{ }_{R} t\right) \times{ }_{R} \Psi^{*}\left({ }_{R} X,{ }_{R} t\right) .
$$

At the end, we obtain

$$
{ }_{R} Y \equiv{ }_{R} \Psi^{*}\left({ }_{R} X,{ }_{R} t\right) .
$$




\section{Quod erat demonstrandum.}

\subsection{Theorem: The Relationship between the (Complex) Number ${ }_{R} S$ and the Complex} Conjugate ${ }_{R} \Psi^{*}\left({ }_{R} X,{ }_{R} t\right)$ of the Wavefunction ${ }_{R} \Psi\left({ }_{R} X,{ }_{R} t\right)$

Claim. (Theorem. Proposition. Statement.)

In general, it is

$$
{ }_{R} \Psi^{*}\left({ }_{R} X,{ }_{R} t\right)=\frac{1}{{ }_{R} S} .
$$

\section{Direct proof.}

In general, axiom I is determined as

$$
+1=+1 .
$$

Multiplying this equation by ${ }_{R} Y$, we obtain

$$
{ }_{R} Y \times 1={ }_{R} Y \times 1 .
$$

Due to Equation (148), it is ${ }_{R} Y={ }_{R} \Psi^{*}\left({ }_{R} X,{ }_{R} t\right)$. Consequently, substituting this equation into Equation (151) we obtain

$$
{ }_{R} \Psi^{*}\left({ }_{R} X,{ }_{R} t\right)={ }_{R} Y .
$$

Due to Equation (143), it is ${ }_{R} Y=1 /{ }_{R} S$. Consequently, substituting this equation into Equation (152) we obtain

$$
{ }_{R} \Psi^{*}\left({ }_{R} X,{ }_{R} t\right)=\frac{1}{{ }_{R} S} \text {. }
$$

\section{Quod erat demonstrandum.}

\subsection{Theorem: The Probability $1-{ }_{R} p\left({ }_{R} \Psi\left({ }_{R} X,{ }_{R} t\right)\right)$ as Associated with the Hamiltonian ${ }_{R} H$}

Claim. (Theorem. Proposition. Statement.)

The probability $1_{R} p\left({ }_{R} \Psi\left({ }_{R} X,{ }_{R} t\right)\right)$ as associated with the Hamiltonian ${ }_{R} H$ is determined as

$$
1-{ }_{R} p\left({ }_{R} \Psi\left({ }_{R} X,{ }_{R} t\right)\right)={ }_{R} H \times{ }_{R} \Psi^{*}\left({ }_{R} X,{ }_{R} t\right) .
$$

Direct proof.

In general, axiom I is determined as

$$
+1=+1 .
$$

Multiplying Equation (155) by ${ }_{R} S$, we obtain

$$
{ }_{R} S \times 1={ }_{R} S \times 1 .
$$

Due to our definition above, we obtain

$$
{ }_{R} H+{ }_{R} \Psi\left({ }_{R} X,{ }_{R} t\right)={ }_{R} S .
$$

Multiplying Equation (157) by the complex conjugate of the wave function ${ }_{R} \Psi^{*}\left({ }_{R} X,{ }_{R} t\right)$, it is

$$
{ }_{R} H \times{ }_{R} \Psi^{*}\left({ }_{R} X,{ }_{R} t\right)+{ }_{R} \Psi\left({ }_{R} X,{ }_{R} t\right) \times{ }_{R} \Psi^{*}\left({ }_{R} X,{ }_{R} t\right)={ }_{R} S \times{ }_{R} \Psi^{*}\left({ }_{R} X,{ }_{R} t\right) .
$$

Due to Equation (153) it is $\left.{ }_{R} S \times{ }_{R} \Psi^{*}{ }_{R} X,{ }_{R} t\right)=1$. Thus far, Equation (158) changes to

$$
{ }_{R} H \times{ }_{R} \Psi^{*}\left({ }_{R} X,{ }_{R} t\right)+{ }_{R} \Psi\left({ }_{R} X,{ }_{R} t\right) \times{ }_{R} \Psi^{*}\left({ }_{R} X,{ }_{R} t\right)=1 .
$$

Following Born's rule, it is ${ }_{R} p\left({ }_{R} \Psi\left({ }_{R} X,{ }_{R} t\right)\right)={ }_{R} \Psi\left({ }_{R} X,{ }_{R} t\right) \times{ }_{R} \Psi^{*}\left({ }_{R} X,{ }_{R} t\right)$. We obtain 


$$
{ }_{R} H \times{ }_{R} \Psi^{*}\left({ }_{R} X,{ }_{R} t\right)+{ }_{R} p\left({ }_{R} \Psi\left({ }_{R} X,{ }_{R} t\right)\right)=1 .
$$

At the end, it is

$$
1-{ }_{R} p\left({ }_{R} \Psi\left({ }_{R} X,{ }_{R} t\right)\right)={ }_{R} H \times{ }_{R} \Psi^{*}\left({ }_{R} X,{ }_{R} t\right) .
$$

\section{Quod erat demonstrandum.}

\subsection{Theorem: The Normalization of the Relationship between Energy and Time}

Claim. (Theorem. Proposition. Statement.)

The relationship between Energy ${ }_{R} E$ and time ${ }_{R} t$ can be normalized as

$$
\frac{{ }_{R} E}{{ }_{R} S}+\frac{{ }_{R} t}{{ }_{R} S}=+1 .
$$

\section{Direct proof.}

In general, axiom I is determined as

$$
+1=+1 \text {. }
$$

Multiplying Equation (163) by ${ }_{R} S$, we obtain

$$
{ }_{R} S={ }_{R} S
$$

Due to our definition, we obtain

$$
{ }_{R} E+{ }_{R} t={ }_{R} S .
$$

We divide the Equation (165) by ${ }_{R} S$. The relationship between energy and time is normalized to 1 as

$$
\frac{{ }_{R} E}{{ }_{R} S}+\frac{{ }_{R} t}{{ }_{R} S}=+1 .
$$

\section{Quod erat demonstrandum.}

\subsection{Theorem: The Normalization of the Relationship between Matter and Gravitational Field}

In general, the modification of our understanding of space and time undergone through Einstein's relativity theory is indeed a profound one. But even Einstein's relativity theory does not [19] give satisfactory answers to a lot of questions. Einstein's successful geometrization of the gravitational field in his general theory of relativity does not include a geometrized theory of the electromagnetic field too. The theoretical physicists working in the field of the general theory of relativity were not able to succeed in finding a convincing geometrical formulation of the gravitational and electromagnetic field. Still, electromagnetic fields are not described by Riemannian metrics. More serious from the conceptual point of view, in order to achieve unification, with the development of quantum theory any conceptual unification of the gravitational and electromagnetic field should introduce a possibility that the fields can be quantized. In our striving toward unification of the foundations of physics a relativistic field theory we are looking for should therefore be an extension of the general theory of relativity and equally and of no less importance a generalization of the theory of the gravitational field. Evidently, following up these train of thoughts and in view of all these difficulties, the following theorem is based on a (gravitational) field of more complex nature. Still, in our attempt to obtain a deeper knowledge of the foundations of physics the new and basic concepts are in accordance with general relativity theory from the beginning but with philosophy too. The purpose of this theorem is to provide some new and basic fundamental insights of the relationship between matter and the gravitational field.

Claim. (Theorem. Proposition. Statement.)

The relationship between the quantum mechanical operator of matter and the wavefunction of the gravitational field can be normalized as 


$$
\frac{{ }_{R} G}{{ }_{R} U}+\frac{{ }_{R} M}{{ }_{R} U}=+1 \text {. }
$$

Direct proof.

In general, axiom I is determined as

$$
+1=+1 .
$$

Multiplying Equation (168) by ${ }_{R} U$, we obtain

$$
{ }_{R} U={ }_{R} U
$$

which is equivalent to

$$
{ }_{R} U+0={ }_{R} U \text {. }
$$

In general it is ${ }_{R} M-{ }_{R} M=0$. In our understanding ${ }_{R} M$ is a determining part of ${ }_{R} U$. Thus far, we obtain

$$
{ }_{R} U-{ }_{R} M+{ }_{R} M={ }_{R} U \text {. }
$$

Following, Einstein all but matter is gravitational field. We obtain ${ }_{R} G={ }_{R} U-{ }_{R} M$. Thus far, it follows that

$$
{ }_{R} G+{ }_{R} M={ }_{R} U \text {. }
$$

We divide the Equation (172) by ${ }_{R} U$. The relationship between the quantum mechanical operator of matter ${ }_{R} M$ and the wavefunction of the gravitational field ${ }_{R} G$ is normalized to 1 as

$$
\frac{{ }_{R} G}{{ }_{R} U}+\frac{{ }_{R} M}{{ }_{R} U}=+1 \text {. }
$$

\section{Quod erat demonstrandum.}

\subsection{Theorem: The Equivalence of Gravitational Field and Time}

Time has always featured prominently discussion in science and is especially associated with the logic of change. A good deal of the scientific work with respect to time has been especially important since the beginning of science as such. Under which conditions are we allowed assuming a period of time during which nothing changes? Further coverage, ahistorical overview and a general presentation of these and other topics related to time as such are available or can be found in literature. A proper discussion of the various views of the nature of the relationship between time and gravitational field and the different issues related to time and gravitational field as such would take us far beyond the scope of this simple theorem. For our purposes, time and gravitational field are related somehow. In any case, especially due to Einstein's relativity theory, there is a very close relationship between time the gravitational field and vice versa. According to this line of thought, we will analyze the relationship between time and gravitational field from the standpoint of Einstein's theory of special relativity. Questions about the nature of time appear to be closely connected to the issue of gravitational field itself. Under conditions of special theory of relativity, clocks which are moving with respect to an inertial system of observation are found to be running more slowly. A clock which is closer to the gravitational mass (i.e. deeper in its "gravity well") appears to go more slowly than a clock which is more distant from the same mass (energy). For example, clocks on the former Space Shuttle where found to ran slightly slower than reference clocks on the Earth. Thus far, the stronger the gravitational potential (i.e. the closer a clock is to the source of the gravitational field, the slower time passes). The gravitational time dilation has been repeatedly confirmed experimentally by several tests of general relativity. Altogether, the behavior of time as such is linked to the behavior of gravitational field and vice versa even if we still don't know how. The aim of this theorem is to work out the interior logic between gravitational field and time. As we will see, the relationship between time and gravitational field is similar to the relationship between mass and energy or the gravitational field is equivalent to time and vice versa, both are equivalent [20] or identical. Finally, we must accept the gravitational field-time equivalence.

Claim. (Theorem. Proposition. Statement.)

The gravitational field and time are equivalent. In general, it is 


$$
{ }_{R} G \equiv \frac{{ }_{R} t}{c^{2}} .
$$

\section{Direct proof.}

In general, axiom I is determined as

$$
+1=+1 \text {. }
$$

Due to Equation (166), it is

$$
+1=\frac{{ }_{R} E}{{ }_{R} S}+\frac{{ }_{R} t}{{ }_{R} S} .
$$

Due to Equation (173), Equation (176) changes to

$$
\frac{{ }_{R} G}{{ }_{R} U}+\frac{{ }_{R} M}{{ }_{R} U}=\frac{{ }_{R} E}{{ }_{R} S}+\frac{{ }_{R} t}{{ }_{R} S} .
$$

Multiplying this equation by ${ }_{R} U$, it is

$$
{ }_{R} G+{ }_{R} M=\frac{{ }_{R} U}{{ }_{R} S} \times{ }_{R} E+\frac{{ }_{R} U}{{ }_{R} S} \times{ }_{R} t .
$$

According to our definition, it is ${ }_{R} S=c^{2} \times{ }_{R} U$. Thus far, it is $1 / c^{2}={ }_{R} U /{ }_{R} S$. Equation (178) changes to

$$
{ }_{R} G+{ }_{R} M=\frac{{ }_{R} E}{c^{2}}+\frac{{ }_{R} t}{c^{2}} .
$$

Due to our definition of matter as ${ }_{R} M={ }_{R} E / c^{2}$. Equation (179) changes to

$$
{ }_{R} G+{ }_{R} M={ }_{R} M+\frac{{ }_{R} t}{C^{2}} .
$$

The matter term ${ }_{R} M$ drops out, and what is left is the wavefunction of the gravitational field ${ }_{R} G$ as

$$
{ }_{R} G=\frac{{ }_{R} t}{c^{2}}
$$

\section{Quod erat demonstrandum.}

\subsection{Theorem: The Normalization of the Relationship between the Hamitonian and the Wavefunction}

Claim. (Theorem. Proposition. Statement.)

The relationship between the Hamiltonian operator and the wavefunction can be normalized as

$$
\frac{{ }_{R} H}{{ }_{R} S}+\frac{{ }_{R} \Psi\left({ }_{R} X,{ }_{R} t\right)}{{ }_{R} S}=+1 .
$$

Direct proof.

In general, axiom I is determined as

$$
+1=+1 \text {. }
$$

Multiplying Equation (183) by ${ }_{R} S$, we obtain

$$
{ }_{R} S={ }_{R} S .
$$

Due to our definition, we obtain

$$
{ }_{R} H+{ }_{R} \Psi\left({ }_{R} X,{ }_{R} t\right)={ }_{R} S .
$$


Divide Equation (185) by ${ }_{R} S$. The normalization of the relationship between the Hamiltonian and the wavefunction follows as

$$
\frac{{ }_{R} H}{{ }_{R} S}+\frac{{ }_{R} \Psi\left({ }_{R} X,{ }_{R} t\right)}{{ }_{R} S}=+1 .
$$

\section{Quod erat demonstrandum.}

\subsection{Theorem: The Wavefunction of the Gravitational Field ${ }_{R} G$}

The hottest and one of the major unresolved problems of today's quantum mechanics is the physical meaning of the wave function. The debate about the physical meaning of the wave function raises broader issues as well. In brief, the difficulties stemmed from an apparent conflict about the existence of an objective reality existing independent of the human mind and consciousness. The purpose of this theorem is to investigate the meaning of the wave function by analyzing the relationship between the wave function and the wave function of the gravitational field itself. As we will see, the wavefunction of a system and the wave function of the gravitational of the same system are equivalent.

Claim. (Theorem. Proposition. Statement.)

In general, the wavefunction of the gravitational field ${ }_{R} G$ is determined as

$$
{ }_{R} G \equiv \frac{{ }_{R} \Psi\left({ }_{R} X,{ }_{R} t\right)}{c^{2}} .
$$

\section{Direct proof.}

In general, axiom I is determined as

$$
+1=+1 \text {. }
$$

Due to Equation (186), it is

$$
+1=\frac{{ }_{R} H}{{ }_{R} S}+\frac{{ }_{R} \Psi\left({ }_{R} X,{ }_{R} t\right)}{{ }_{R} S} .
$$

Due Equation (173), Equation (189) changes to

$$
\frac{{ }_{R} G}{{ }_{R} U}+\frac{{ }_{R} M}{{ }_{R} U}=\frac{{ }_{R} H}{{ }_{R} S}+\frac{{ }_{R} \Psi\left({ }_{R} X,{ }_{R} t\right)}{{ }_{R} S} .
$$

Multiplying this equation by ${ }_{R} U$, it is

$$
{ }_{R} G+{ }_{R} M=\frac{{ }_{R} U}{{ }_{R} S} \times{ }_{R} H+\frac{{ }_{R} U}{{ }_{R} S} \times{ }_{R} \Psi\left({ }_{R} X,{ }_{R} t\right) .
$$

According to our definition, it is ${ }_{R} S=c^{2} \times{ }_{R} U$. Thus far, it is $1 / c^{2}={ }_{R} U /{ }_{R} S$. Equation (191) changes to

$$
{ }_{R} G+{ }_{R} M=\frac{{ }_{R} H}{c^{2}}+\frac{{ }_{R} \Psi\left({ }_{R} X,{ }_{R} t\right)}{c^{2}} .
$$

Due to our definition of matter as ${ }_{R} M={ }_{R} H / c^{2}$, Equation (192) changes to

$$
{ }_{R} G+{ }_{R} M={ }_{R} M+\frac{{ }_{R} \Psi\left({ }_{R} X,{ }_{R} t\right)}{c^{2}} .
$$

Subtracting ${ }_{R} M$ on both sides of the equation before, the wavefunction of the gravitational field ${ }_{R} G$ follows that

$$
{ }_{R} G=\frac{{ }_{R} \Psi\left({ }_{R} X,{ }_{R} t\right)}{c^{2}} .
$$

Quod erat demonstrandum. 


\subsection{Theorem: The Equivalence of Time and Wavefunction}

The (quantum mechanical) system, and with it the wavefunction varies with time. Theoretically, in this sense the wave function can be regarded as a function of time also. In general, the actual dependence of the wave function on time is determined by equations thus that the wave function ${ }_{R} \Psi\left({ }_{R} X,{ }_{R} t\right)$ and the time ${ }_{R} t$ as measured by a stationary observer $R$ are equivalent.

Claim. (Theorem. Proposition. Statement.)

Under conditions of the special theory of relativity from the standpoint of a stationary observer $R$ it is

$$
{ }_{R} t={ }_{R} \Psi\left({ }_{R} X,{ }_{R} t\right) .
$$

Direct proof.

In general, axiom $\mathrm{I}$ is determined as

$$
+1=+1 .
$$

Multiplying this equation by ${ }_{R} G$, the wave function of the gravitational field, we obtain

$$
{ }_{R} G \times 1={ }_{R} G \times 1 .
$$

Due to Equation (181), it is ${ }_{R} G={ }_{R} t / c^{2}$. We obtain

$$
\frac{{ }_{R} t}{C^{2}}={ }_{R} G
$$

According to Equation (194), $t$ is ${ }_{R} G={ }_{R} \Psi\left({ }_{R} X,{ }_{R} t\right) / c^{2}$. Rearranging equation, we obtain

$$
\frac{{ }_{R} t}{c^{2}}=\frac{{ }_{R} \Psi\left({ }_{R} X,{ }_{R} t\right)}{c^{2}} .
$$

Rearranging Equation (199), we obtain

$$
{ }_{R} t={ }_{R} \Psi\left({ }_{R} X,{ }_{R} t\right) .
$$

\section{Quod erat demonstrandum.}

\section{Scholium.}

Any state of a system at a given moment can be described by a definite (in general complex) wave function (sometimes also the probability amplitude). The square of the modulus of this wave function determines the probability of the various results of any other measurement of a system. The sum of the probabilities of all possible values of a system must, by definition, must be equal to unity. It is therefore necessary that the result of integrating $|\Psi|^{2}$ should be equal to unity too (the normalisation condition for wave function). However, it is possible to use wave functions which are not normalized. In any physical system with waves, the waveform at a given time is a function of the source. In quantum mechanics, a wave can be described by a wave function. Moreover, the chief positive principle of quantum mechanics called the principle of superposition demands that a wave function is determined by a superposition (called “quantum superposition”) of (possibly infinitely many) other wave functions of a certain type (called "eigenfunction”). The probability for a specified configuration is given by the square of the absolute value of a complex coefficient.

\subsection{Theorem: The Relationship between Energy ${ }_{R} E$, Time ${ }_{R} t$ and Space ${ }_{R} S$}

Claim. (Theorem. Proposition. Statement.)

In general, it is

$$
{ }_{R} E+{ }_{R} t={ }_{R} S \text {. }
$$

Direct proof.

In general, axiom I is determined as

$$
+1=+1 \text {. }
$$


Multiplying this equation by $\left({ }_{R} H+{ }_{R} \Psi\left({ }_{R} X,{ }_{R} t\right)\right)$ we obtain

$$
\left({ }_{R} H+{ }_{R} \Psi\left({ }_{R} X,{ }_{R} t\right)\right) \times 1=\left({ }_{R} H+{ }_{R} \Psi\left({ }_{R} X,{ }_{R} t\right)\right) \times 1 .
$$

Due to our definition of ${ }_{R} S=\left({ }_{R} H+{ }_{R} \Psi\left({ }_{R} X,{ }_{R} t\right)\right)$, Equation (203) changes to

$$
{ }_{R} H+{ }_{R} \Psi\left({ }_{R} X,{ }_{R} t\right)={ }_{R} S .
$$

In our understanding, it is ${ }_{R} H={ }_{R} E$. Substituting this relationship into Equation (204) it follows that

$$
{ }_{R} E+{ }_{R} \Psi\left({ }_{R} X,{ }_{R} t\right)={ }_{R} S .
$$

Due to Equation (200) it is ${ }_{R} t={ }_{R} \Psi\left({ }_{R} X,{ }_{R} t\right)$. Substituting this relationship into Equation (205) it follows in general that

$$
{ }_{R} E+{ }_{R} t={ }_{R} S .
$$

Quod erat demonstrandum.

\subsection{Theorem: The Normalization of the Relationship between the Gravitational Field $o g$ and the Gravitational Wave wg}

Claim. (Theorem. Proposition. Statement.)

In general, it is

$$
\frac{{ }_{o} g^{2}}{{ }_{R} g^{2}}+\frac{{ }_{w} g^{2}}{{ }_{R} g^{2}}=1
$$

Direct proof.

In general, axiom I is determined as

$$
+1=+1 .
$$

Multiplying Equation (207) by $\left(\left(o^{2} / t^{2}\right)+\left(v^{2} / c^{2}\right)\right)$, we obtain

$$
\left(\frac{{ }_{O} t^{2}}{{ }_{R} t^{2}}+\frac{v^{2}}{c^{2}}\right) \times 1 \equiv\left(\frac{{ }_{O} t^{2}}{{ }_{R} t^{2}}+\frac{v^{2}}{c^{2}}\right) \times 1 .
$$

According to the relationship $\left(\left(o^{2} /{ }_{R} t^{2}\right)+\left(v^{2} / c^{2}\right)\right)=1$, Equation (208) simplifies as

$$
\left(\frac{{ }_{O} t^{2}}{{ }_{R} t^{2}}+\frac{v^{2}}{c^{2}}\right)=1 \text {. }
$$

Equation (209) can be rearranged as

$$
\frac{{ }_{0} t^{2} \times c^{2} \times c^{2}}{{ }_{R} t^{2} \times c^{2} \times c^{2}}+\frac{v^{2}}{c^{2}}=1 .
$$

and equally as

$$
\frac{{ }_{0} t^{2} \times c^{2} \times c^{2}}{c^{2} \times c^{2} \times{ }_{R} t^{2}}+\frac{v^{2} \times c^{2} \times{ }_{R} g^{2}}{c^{2} \times c^{2} \times{ }_{R} g^{2}}=1 .
$$

Due to our definition it is ${ }_{o} g={ }_{O} t / c^{2}$ and equally ${ }_{R} g={ }_{R} t / c^{2}$, Equation (211) simplifies as to

$$
\frac{{ }_{o} g^{2}}{{ }_{R} g^{2}}+\frac{v^{2} \times c^{2} \times{ }_{R} g^{2}}{c^{2} \times c^{2} \times{ }_{R} g^{2}}=1 \text {. }
$$

According to our definition it is $w_{w}=v \times c \times{ }_{R} g$ and thus far $t^{2} t^{2}=\left(v \times c \times{ }_{R} g\right)^{2}$. Equation (212) changes to 


$$
\frac{{ }_{0} g^{2}}{{ }_{R} g^{2}}+\frac{{ }_{W} t^{2}}{c^{2} \times c^{2} \times{ }_{R} g^{2}}=1 .
$$

The definition of the gravitational waves was $w_{w}={ }_{w} t / c^{2}$. Thus far, it is $w_{w} g^{2}\left({ }_{w} t / c^{2}\right)^{2}$. The normalization of the relationship between the gravitational field $o g$ and the gravitational waves ${ }_{w} g$ follows as

$$
\frac{{ }_{O} g^{2}}{{ }_{R} g^{2}}+\frac{{ }_{W} g^{2}}{{ }_{R} g^{2}}=1 \text {. }
$$

\section{Quod erat demonstrandum.}

\section{Scholium.}

This theorem leads to some consequences. The relationship

$$
\frac{{ }_{0} g^{2}}{{ }_{R} g^{2}}+\frac{{ }_{W} g^{2}}{{ }_{R} g^{2}}=1
$$

is equivalent with the relationship

$$
\frac{{ }_{O} t^{2}}{{ }_{R} t^{2}}+\frac{{ }_{W} g^{2} \times c^{2} \times c^{2}}{{ }_{R} t^{2}}=1
$$

and thus far with the relation

$$
\frac{{ }_{W} g^{2} \times c^{2} \times c^{2}}{{ }_{R} t^{2}}=\left(1-\frac{{ }_{O} t^{2}}{{ }_{R} t^{2}}\right)
$$

or with

$$
{ }_{W} g^{2}=\frac{{ }_{R} t^{2}}{c^{2} \times c^{2}} \times\left(1-\frac{{ }_{O} t^{2}}{{ }_{R} t^{2}}\right) .
$$

Due to special relativity it is $\left(\left({ }_{o} t^{2} /{ }_{R} t^{2}\right)+\left(v^{2} / c^{2}\right)\right)=1$ and thus far $\left(v^{2} / c^{2}\right)=1-\left({ }_{o} t^{2} /{ }_{R} t^{2}\right)$. We obtain

$$
{ }_{W} g^{2}=\frac{{ }_{R} t^{2}}{c^{2} \times c^{2}} \times \frac{v^{2}}{c^{2}} .
$$

As we will see later, the quantum-mechanical wave function ${ }_{R} \Psi\left({ }_{R} X,{ }_{R} t\right)$ and the time ${ }_{R} t$ are identical. Consequently, the quantum-mechanical wave function ${ }_{R} \Psi\left({ }_{R} X,{ }_{R} t\right)$ can be used to describe the gravitational waves ${ }_{W} g$ as

$$
{ }_{W} g^{2}=\frac{v^{2}}{c^{2} \times c^{2} \times c^{2}} \times{ }_{R} \Psi\left({ }_{R} X,{ }_{R} t\right) \times{ }_{R} \Psi\left({ }_{R} X,{ }_{R} t\right) .
$$

\subsection{Theorem: The Quantization of the Gravitational Field}

The current understanding of gravitation and the gravitational field is based on Albert Einstein's general theory of relativity. In contrast to Einstein's general relativity, all non-gravitational forces are described within the framework of quantum mechanics. Clearly there is something unsatisfactory about the framework of quantum mechanics, a quantum mechanical description of gravitation and the gravitational field consistent with Einstein's theory of relativity is still not in sight. More and more, one often gets the impression that we are far away from the verge of a Theory of Everything and that combining gravity with all the others fundamental forces of nature is much more than just a matter of technical details.

From a technical point of view, the conceptual and technical problems associated with the approaches to quantize the gravitational field, known in the literature as "covariant" (particle physics) and "canonical” quantum gravity, disabled until today to construct a theory in which the gravitational field is treated quantummechanically. As a result, many theorists have taken up more radical approaches to the problem of the quantization of the gravitational field, quantum gravity, the most popular approaches being loop quantum gravity and 
string theory. This publication makes no pretence to be either complete or up-to-date of today's most popular approaches of the quantization of the gravitational field. A review of the contemporary methods of quantization of the gravitational field will not be given. The goal of this theorem is to follow a completely new approach and to solve the problem of the quantization of the gravitational field. To do this and to reconcile general relativity with the principles of quantum mechanics, we followed strictly Einstein's understanding of the relationship between matter and gravitational field.

Claim. (Theorem. Proposition. Statement.)

In general, the quantization of the gravitational field is determined by the equation

$$
{ }_{R} M \times{ }_{R} G=\frac{i \hbar}{c^{2}} \frac{\partial}{\partial t} \frac{{ }_{R} \Psi\left({ }_{R} X,{ }_{R} t\right)}{c^{2}} .
$$

\section{Direct proof.}

In general, axiom I is determined as

$$
+1=+1 \text {. }
$$

Multiplying the Schrödinger equation, we obtain

$$
{ }_{R} H \times{ }_{R} \Psi\left({ }_{R} X,{ }_{R} t\right) \times 1={ }_{R} H \times{ }_{R} \Psi\left({ }_{R} X,{ }_{R} t\right) \times 1 .
$$

or equally

$$
{ }_{R} H \times{ }_{R} \Psi\left({ }_{R} X,{ }_{R} t\right)=i \hbar \frac{\partial}{\partial t}{ }_{R} \Psi\left({ }_{R} X,{ }_{R} t\right) .
$$

Dividing by the speed of the light squared, we obtain

$$
\frac{{ }_{R} H}{c^{2}} \times \frac{{ }_{R} \Psi\left({ }_{R} X,{ }_{R} t\right)}{c^{2}}=\frac{i \hbar}{c^{2}} \frac{\partial}{\partial t} \frac{{ }_{R} \Psi\left({ }_{R} X,{ }_{R} t\right)}{c^{2}} .
$$

Due to our definition of matter it is ${ }_{R} M={ }_{R} H / c^{2}$. The equation before changes to

$$
{ }_{R} M \times \frac{{ }_{R} \Psi\left({ }_{R} X,{ }_{R} t\right)}{c^{2}}=\frac{i \hbar}{c^{2}} \frac{\partial}{\partial t} \frac{{ }_{R} \Psi\left({ }_{R} X,{ }_{R} t\right)}{c^{2}}
$$

Due to Equation (194) it is ${ }_{R} G={ }_{R} \Psi\left({ }_{R} X,{ }_{R} t\right) / c^{2}$. The quantization of the gravitational field follows as

$$
{ }_{R} M \times{ }_{R} G=\frac{i \hbar}{c^{2}} \frac{\partial}{\partial t} \frac{{ }_{R} \Psi\left({ }_{R} X,{ }_{R} t\right)}{c^{2}} .
$$

\section{Quod erat demonstrandum.}

\section{Scholium.}

Thus far, quantum mechanics strictly predicts that all matter is quantum while general relativity describes the gravitational effects of classical matter. Then again, one might argue that we cannot reconcile general relativity with the principles of quantum mechanics at all. In view of many possible approaches to quantize the gravitational field already developed, we described the dynamics of the gravitational field by the principles of quantum mechanics while following strictly the rules of Einstein's theory of relativity.

\subsection{Theorem: The Physical Meaning of the Wavefunction}

Conceptual difficulties are associated with quantum mechanics since its inception, despite of quantum mechanics extraordinary predictive successes. One "defining” part of quantum mechanics is the Schrödinger's equation. Quite naturally, Schrödinger's equation is to quantum mechanics what Newton's second law of motion is to classical mechanics. A determining part of Schrödinger's equation is the wave function. The nice fact is that quantum mechanics with its revolutionary implications meets still with serious difficulties in telling us what is the physical meaning of the wave function. As already stated, reconsidering basic concepts of one of the most successful theories in the history of human science, a very natural question which all scientists who are concerned about the physical meaning of the wave function is, what could be the correct starting point to solve this 
problem. This theorem will overcome the difficulties about the physical meaning of the wave function posed by quantum mechanics by strictly following Einstein in his proposal of to accept a physical reality as a kind of objective reality, a physical reality which is independent of the human being, his consciousness and his mind. The purpose of the present theorem is not to put forward a new and complete self-consistent interpretation of quantum mechanics which is able fully to highlight the explicative power of this revolutionary theory. But to fully appreciate the novel aspects contained in this theorem, it is more than necessary and highly useful to recall, clarify and elaborate about some basic notions needed to solve the problem of the physical meaning of the wave function. To complete this short overview, it is straightforward to convince oneself that the Hamiltonian, usually denoted by $H$ or as ${ }_{R} H$, is a quantum mechanical operator corresponding to the total energy of a (quantum mechanical) system. In most of the cases, the spectrum of the Hamiltonian $H$ is the set of the possible outcomes when one measures the total energy of a system. In special relativity, the total energy of the system is denoted by ${ }_{R} E$ and is equally the set of possible outcomes at least ${ }_{O} E+{ }_{\Delta} E={ }_{O} E+{ }_{\underline{O}} \underline{E}={ }_{R} E$.

Claim. (Theorem. Proposition. Statement.)

Under conditions of the special theory of relativity from the standpoint of a stationary observer $R$ it is

$$
{ }_{R} t={ }_{R} \Psi\left({ }_{R} X,{ }_{R} t\right) .
$$

\section{Direct proof.}

In general, axiom I is determined as

$$
+1=+1 .
$$

Multiplying this equation by ${ }_{R} E \times{ }_{R} t$, we obtain

$$
{ }_{R} E \times{ }_{R} t={ }_{R} E \times{ }_{R} t .
$$

The system analyzed by us is completely described by this equation. The same system, no matter how complicated, is equally completely described by Schrödinger equation too. We equate both equations.

$$
{ }_{R} E \times{ }_{R} t={ }_{R} H \times{ }_{R} \Psi\left({ }_{R} X,{ }_{R} t\right) .
$$

The Hamiltonian is a quantum mechanical equivalent of the total energy of a system, the set of all possible outcomes. In special relativity, the total energy of the system is denoted by ${ }_{R} E$. Therefore, we equate both notions as ${ }_{R} E={ }_{R} H$. Rearranging the equation above, it follows that

$$
{ }_{R} E \times{ }_{R} t={ }_{R} E \times{ }_{R} \Psi\left({ }_{R} X,{ }_{R} t\right) .
$$

Finally, our proof is complete. In general, it is

$$
{ }_{R} t={ }_{R} \Psi\left({ }_{R} X,{ }_{R} t\right) .
$$

Quod erat demonstrandum.

\section{Scholium.}

The general requirements of consistency and unity of a theorem assumes of course the absence of contradictions with experiment. The core of the Copenhagen Interpretation of quantum mechanics is the reduction of the wave function which is linked to the measurement processes. Due to Copenhagen Interpretation of quantum mechanics, during the measurement process, the quantum-mechanical wave function ${ }_{R} \Psi\left({ }_{R} X,{ }_{R} t\right)$ reduces to an eigenfunction of the system. Consequently, due to the Copenhagen Interpretation of quantum mechanics the wave function does not possess any real physical meaning. However, questions of this kind deal with only one aspect of quantum theory. To explain the main lines of thought, on this point opinions seem to be divided. In opposite to the canonical Copenhagen Interpretation of quantum mechanics, there are already interpretations of quantum mechanics which attributed a real physical meaning to the wave function. In contrast to the more or less hidden agenda of scientists blocking progress toward a unification of the incompatible descriptions of reality provided by relativity and quantum theory a realistic description of reality at its most fundamental level is possible in principle and necessary.

Especially David Bohm (1917-1992), a theoretical U. S. physicist, rediscovered an interpretation of the quantum theory made by de Broglie [21] in 1926 (de Broglie’s pilot-wave theory), but later given up by him, and presented a principal alternative to the very dogmatic worldview as pushed by the Copenhagen interpretation of 
quantum mechanics. Bohm's nonrelativistic interpretation of quantum mechanics [22], known as "hidden variable" theory, is still held at a distance by most physicists. In the Bohmian mechanical version of nonrelativistic quantum theory, the wave function, obeying Schrödinger's equation, does not provide a complete description of a quantum system. Still, in Bohm's nonrelativistic interpretation of quantum mechanics the wave function gets a real physical meaning. "In contrast to the usual interpretation, this alternative interpretation permits us to conceive of each individual system as being in a precisely definable state, whose changes with time are determined by definite laws, analogous to (but not identical with) the classical equations of motion." [23]. Not long after second world war (1939-1945) Bohm was charged before the Committee on Un-American Activities in May and June 1949, but refused to cooperate with McCarthy's committee. As a consequence, Princeton University fired him, Bohm academic career was derailed. In 1600, the Roman Catholic Church burned Giordano Bruno (1548-1600) alive. In the 1950s the U. S. Administration blacklisted David Bohm by U.S. universities and effectively exiled him to Brazil, Israel and finally to England. On the first sight, it is possible to claim that ${ }_{R} t$ has nothing in common with ${ }_{R} \Psi\left({ }_{R} X,{ }_{R} t\right)$. This relationship can be rearranged something as

$$
{ }_{R} \Psi\left({ }_{R} X,{ }_{R} t\right)={ }_{R} t \times \frac{1}{1}=\frac{{ }_{R} t}{\mathrm{e}^{-\frac{E \times t}{\hbar}}} \times \mathrm{e}^{-\frac{E \times t}{\hbar}} \equiv A \times \mathrm{e}^{-\frac{E \times t}{\hbar}} .
$$

In the above equation we defined $A={ }_{R} t /\left(\mathrm{e}^{-(\ldots)}\right)$. At the end we obtain something like

$$
{ }_{R} \Psi\left({ }_{R} X,{ }_{R} t\right) \equiv A \times \mathrm{e}^{-\frac{E \times t}{\hbar}} .
$$

Thus far, all the changes above have no influence on the fact that ${ }_{R} t={ }_{R} \Psi\left({ }_{R} X,{ }_{R} t\right)$.

\section{Discussion}

The hegemonic standard a causal Copenhagen Interpretation of quantum mechanics established by a small elite of physicists in the 1920s centers around the uncertainty principle (which is meanwhile refuted [24]-[25]) and opened a very wide door to mysticism, logical fallacies and a wishful thinking especially in quantum physics. The political attitude and the ideology of this small elite of physicists (Niels Bohr, Werner Heisenberg, Max Born and view other) played a major role in the construction of the Copenhagen Interpretation of quantum mechanics in the 1920s. Regardless of observation, the associating ideas, the political attitude or the ideology of physicists following strictly the acausal, indeterministic Copenhagen Interpretation of quantum mechanics objective reality (or the properties of matter) is the way the same is. We still find, years after quantum mechanics inception that quantum mechanics is fundamentally only about observation or results of measurement but not about reality as such. On the basis of more or less the same or similar considerations as those above it is claimed that a deterministic completion or reinterpretation of quantum theory is not possible. Strictly speaking, the wave function is still one of the pillars of quantum mechanics. Especially, due to the Copenhagen Interpretation of quantum mechanics the wave function does not possess any physical meaning. Therefore, the theory quantum mechanics, perhaps the most revolutionary theory in the history of science, still raises innumerable questions to physicists, chemists and philosophers of science.

The guideline for Einstein in his physical research was an objective reality existing independently of human mind and consciousness. In particular Einstein was one of the most famous critics of the Copenhagen Interpretation of quantum mechanics. Einstein has always regarded quantum theory as incomplete [26].

Due to the excellent agreement with an extremely wide range of experiments, the most physicists regarded the objections as raised by Einstein and other as not relevant.

The wave function is existing independently and outside of human mind and consciousness and something objective and real. The wave function is the quantum mechanical equivalent of the time ${ }_{R} t$ of the special theory of relativity as measured by a stationary observer $R$. Thus far, there is nothing mysterious with the wave function. In contrast to the representatives of the Copenhagen interpretation, a realistic interpretation of quantum theory will expel any kind of mysticism from physics.

In particular, there is another crucial aspect of quantum mechanics called the reduction of the state vector (i.e. collapse of the wavefunction). The collapse of the wave function and the correct understanding of the collapse of the wave function addresses several distinct, important and far reaching issues of the foundations of today's 
physics and science as such. Originally, the concept of wavefunction collapse was introduced by Werner Heisenberg in his 1927 paper "Über den anschaulichen Inhalt der quantentheoretischen Kinematic und Mechanik" and later incorporated into the mathematical formalism of quantum mechanics by John von Neumann in his 1932 publication "Mathematische Grundlagen der Quantenmechanik”. In his 1927 paper, Heisenberg writes

"durch die experimentelle Feststellung: 'Zustand m' wählen wir aus der Fülle der verschiedenen Möglichkeiten $\left(c_{n m}\right)$ eine bestimmte: $m$ aus, zerstören aber gleichzeitig, wie nachher erläutert wird, alles, was an Phasenbeziehungen noch in den Größen $c_{n m}$ enthalten war" [27].

It is easy understand the core of this problem. A (relativistic) system evolves in time by the continuous evolution via the Schrödinger equation or some relativistic equivalent. Under appropriate circumstances, the wave function, initially in a superposition of several eigenstates, collapses or reduces to a single eigenstate, that what is measured by a moving observer O. However, after the collapse of the wave function, a physical system is determined or described again by a wave function.

Thus far, the continuous evolution via the Schrödinger equation and the collapse of the wave function are the two basic processes by which quantum systems evolve in time. However, let us focus on the appropriate notion of the collapse of the wave function. Is the collapse of the wavefunction a fundamental and objective physical phenomenon of its own, rather than a non-real theoretical mathematical construct? Does the collapse of the wave function takes any time, the collapse time?

The Copenhagen Interpretation of quantum mechanics is grounded on a self-closing chain of circular hypotheses, which are in principle unverifiable. The hypotheses even if found to be inadequate can be made to agree with experiment by some yet unknown changes in the mathematical formulation of the theory without requiring any fundamental changes in the physical interpretation. Thus far, as long as we accept the usual Copenhagen Interpretation of quantum mechanics, it is impossible to give up this interpretation, even if the same interpretation is proofed to be wrong by some (thought) experiments.

\section{Conclusion}

The wave function is one of the most important concepts of quantum mechanics. Schrödinger himself originally attempted a largely classical interpretation of the wave function as a description of real physical wave. Born's probability interpretation of the wave function operating with a mysterious collapse of the wave function during measurement replaced Schrödinger's view and became the standard interpretation of the wave function today. However, the standard interpretation of the wave function is based on measurement and still unsatisfying in principle. Thus far, it is neither possible nor necessary to interpret the wave function as something objective and physically real. Especially, due to standard Copenhagen interpretation, the wave function does not possess any physical meaning; the wave function is not referring to something physically real. In view of these problems, alternative and realistic interpretations [22]-[23], [28]-[30] of the wave function have been proposed. According to the de Broglie-Bohm theory, the wave function is generally taken as an objective physical field. In this paper, we have demonstrated that a correct local realistic interpretation of the wave function is possible without any contradiction. Especially, the problem of the physical meaning of the wave function is solved. The wave function is the quantum mechanical analogue of the notion time ${ }_{R} t$ as determined by a stationary observer $R$ of the theory of special relativity.

\section{Acknowledgements}

I am very happy to have the opportunity to express my very deep gratitude to the Scientific Committee of the conference "Quantum Theory: from foundations to technologies (QTFT)", Linnaeus University, Sweden, June 8-11, 2015. This paper has been accepted for presentation and was presented at the QFTF conference at Linnaeus University, Sweden, June 8-11, 2015. Moreover, it was a very great honor and privilege for me to discuss privately every topic of this paper in detail and with great pleasure with Dr. Theodor W. Hänsch, Nobel Prize in Physics 2005, at the QFTF conference at Linnaeus University, Sweden, June 8-11, 2015. In particular, Dr. Theodor W. Hänsch pointed out the relation to the de Broglie's “matter” waves, for which I am very grateful.

\section{References}

[1] Newton, I. (1686) Philosophiae Naturalis Principia Mathematica. Pepys, Londini, 12. 
[2] Euler, L. (1752) Découverte d’un nouveau principe de mécanique. Memoires de l'Academie Royal des Sciences, 6, 185-217.

[3] Schrödinger, E. (1926) An Undulatory Theory of the Mechanics of Atoms and Molecules. Physical Review, 28, 1049-1070. http://dx.doi.org/10.1103/PhysRev.28.1049

[4] Einstein, A. (1905) Ist die Trägheit eines Körpers von seinem Energieinhalt abhängig? Annalen der Physik, 323, 641. http://dx.doi.org/10.1002/andp.19053231314

[5] Einstein, A. (1912) Relativität und Gravitation. Erwiderung auf eine Bemerkung von M. Abraham. Annalen der Physik, 343, 1059-1064. http://dx.doi.org/10.1002/andp.19123431014

[6] Einstein, A. (1918) Prinzipielles zur allgemeinen Relativitätstheorie. Annalen der Physik, 360, 241-242. http://dx.doi.org/10.1002/andp.19183600402

[7] Lewis, G.N. and Tolman, R.C. (1909) The Principle of Relativity, and Non-Newtonian Mechanics. Proceedings of the American Academy of Arts and Sciences, 44, 711-724. http://dx.doi.org/10.2307/20022495

[8] Barukčić, I. (2013) The Relativistic Wave Equation. International Journal of Applied Physics and Mathematics, 3, 387-391. http://dx.doi.org/10.7763/IJAPM.2013.V3.242

[9] Einstein, A. (1907) Über das Relativitätsprinzip und die aus demselben gezogenen Folgerungen. Jahrbuch der Radioaktivität und Elektronik, 4, 411-462.

[10] Leibniz, G.W. (1686) Brevis demonstratioerrorismemorabilis Cartesiietaliorum circa legemnaturalem, secundum quam volunt a Deoeandem semper quantitatemmotusconservari, qua et in re mechanicaabuntur. Acta Eruditorum, 3, 161-163.

[11] Leibniz, G.W. (1695) Specimen dynamicum pro admirandis Naturaelegibus circa corporum vires et mutuasactionesdetegendis, et ad suascausasrevocandis. Acta Eruditorum, 4, 145-157.

[12] Einstein, A. (1905) Zur Elektrodynamik bewegter Körper. Annalen der Physik, 322, 891-921. http://dx.doi.org/10.1002/andp.19053221004

[13] Einstein, A. (1916) Die Grundlage der allgemeinen Relativitätstheorie. Annalen der Physik, 354, 802-803. http://dx.doi.org/10.1002/andp.19163540702

[14] Born, M. (1926) Zur Quantenmechanik der Stoßvorgänge. Zeitschriftfür Physik, 37, 363-367. http://dx.doi.org/10.1007/BF01397477

[15] Einstein, A. and de Sitter, W. (1923). On the Relation between the Expansion and the Mean Density of the Universe. Proceedings of the National Academy of Sciences of the United States of America, 18, 213-214. http://dx.doi.org/10.1073/pnas.18.3.213

[16] Einstein, A. (1918) Prinzipielles zur allgemeinen Relativitätstheorie. Annalen der Physik, 360, 241-244. http://dx.doi.org/10.1002/andp.19183600402

[17] Davisson, C. and Germer, L.H. (1927) Diffraction of Electrons by a Crystal of Nickel. Physical Review, 30, 705-740. http://dx.doi.org/10.1103/PhysRev.30.705

[18] Barukčić, I. (2016) Newton’s Gravitational Constant Big G Is Not a Constant. Journal of Modern Physics, 7, 510-522. http://dx.doi.org/10.4236/jmp.2016.76053

[19] Barukčić, I. (2015) Anti Einstein-Refutation of Einstein's General Theory of Relativity. International Journal of Applied Physics and Mathematics, 5, 18-28.

[20] Barukčić, I. (2011) The Equivalence of Time and Gravitational Field. Physics Procedia, 22, 56-62. http://dx.doi.org/10.1016/j.phpro.2011.11.008

[21] de Broglie, L. (1930) An Introduction to the Study of Wave Mechanics. E. P. Dutton and Company, Inc., New York, (See Chap. 6, 9, and 10).

[22] Bohm, D. (1952) A Suggested Interpretation of the Quantum Theory in Terms of 'Hidden' Variables. II. Physcial Review, 85, 180-193. http://dx.doi.org/10.1103/PhysRev.85.180

[23] Bohm, D. (1952) A Suggested Interpretation of the Quantum Theory in Terms of "Hidden” Variables. I. Physcial Review, 85, 166-179. http://dx.doi.org/10.1103/PhysRev.85.166

[24] Barukčić, I. (2011) Anti Heisenberg-Refutation of Heisenberg’s Uncertainty Relation. American Institute of Physics-Conference Proceedings, 1327, 322.

[25] Barukčić, I. (2014) Anti Heisenberg-Refutation of Heisenberg's Uncertainty Principle. International Journal of Applied Physics and Mathematics, 4, 244-250. http://dx.doi.org/10.7763/IJAPM.2014.V4.292

[26] Einstein, A., Podolsky, B. and Rosen, N. (1935) Can Quantum-Mechanical Description of Physical Reality Be Considered Complete? Physical Review, 47, 777. http://dx.doi.org/10.1103/PhysRev.47.777

[27] Heisenberg, W. (1927) Über den anschaulichen Inhalt der quantentheoretischen Kinematik und Mechanik. Zeit- 
schriftfür Physik, 43, 183. http://dx.doi.org/10.1007/BF01397280

[28] Everett, H. (1957) “Relative State” Formulation of Quantum Mechanics. Reviews of Modern Physics, 29, 454-462. http://dx.doi.org/10.1103/RevModPhys.29.454

[29] Nelson, E. (1966) Derivation of the Schrödinger Equation from Newtonian Mechanics. Physical Review, 150, 10791085. http://dx.doi.org/10.1103/PhysRev.150.1079

[30] Ghirardi, G.C., Grassi, R. and Benatti, F. (1995) Describing the Macroscopic World: Closing the Circle within the Dynamical Reduction Program. Foundations of Physics, 25, 5-38. http://dx.doi.org/10.1007/BF02054655 